\title{
Steady-State Electrical Conduction in the Periodic Lorentz Gas
}

(C) Springer-Verlag 1993

\author{
N.I. Chernov ${ }^{1,2}$, G.L. Eyink ${ }^{3}$, J.L. Lebowitz ${ }^{3}$, and Ya.G. Sinai ${ }^{4}$ \\ ${ }^{1}$ Center for Dynamical Systems and Nonlinear Studies, Georgia Institute of Technology, \\ Atlanta, Ga 30332, USA \\ 2 Joint Institute for Nuclear Research, Dubna, P.O. Box 79, Head Post Office, Moscow, Russia \\ ${ }^{3}$ Departments of Mathematics and Physics, Rutgers University, New Brunswick, NJ 08903, \\ USA \\ ${ }^{4}$ L.D. Landau Institute for Theoretical Physics, Russian Academy of Sciences, 117940 GSP-1 \\ Moscow V-334, Russia
}

Received December 2, 1992

Dedicated to Elliott Lieb

\begin{abstract}
We study nonequilibrium steady states in the Lorentz gas of periodic scatterers when an electric external field is applied and the particle kinetic energy is held fixed by a "thermostat" constructed according to Gauss' principle of least constraint (a model problem previously studied numerically by Moran and Hoover). The resulting dynamics is reversible and deterministic, but does not preserve Liouville measure. For a sufficiently small field, we prove the following results: (1) existence of a unique stationary, ergodic measure obtained by forward evolution of initial absolutely continuous distributions, for which the Pesin entropy formula and Young's expression for the fractal dimension are valid; (2) exact identity of the steady-state thermodynamic entropy production, the asymptotic decay of the Gibbs entropy for the time-evolved distribution, and minus the sum of the Lyapunov exponents; (3) an explicit expression for the full nonlinear current response (Kawasaki formula); and (4) validity of linear response theory and Ohm's transport law, including the Einstein relation between conductivity and diffusion matrices. Results (2) and (4) yield also a direct relation between Lyapunov exponents and zero-field transport ( $=$ diffusion) coefficients. Although we restrict ourselves here to dimension $d=2$, the results carry over to higher dimensions and to some other physical situations: e.g. with additional external magnetic fields. The proofs use a well-developed theory of small perturbations of hyperbolic dynamical systems and the method of Markov sieves, an approximation of Markov partitions.
\end{abstract}

\section{Physical Discussion and Statement of Results}

(a) Introduction. We consider in this paper a dynamical system which corresponds to the motion of a single particle between a finite number of fixed, disjoint, convex scatterers in a periodic domain of the plane $\mathbb{R}^{2}$. As in the previous works $[2,3]$, the particle changes its velocity at moments of collision according to the usual law of elastic reflection, but, unlike there, the particle motion between collisions is not the free one at constant velocity. Instead, the motion between collisions is governed by 
the following set of first-order equations:

$$
\begin{aligned}
& \dot{\mathbf{q}}=\mathbf{p} / m, \\
& \dot{\mathbf{p}}=\mathbf{E}-\zeta \cdot \mathbf{p} .
\end{aligned}
$$

Here, $\mathbf{q}=\left(q_{1}, q_{2}\right)$ are the Cartesian coordinates of the particle, and $\mathbf{p}=\left(p_{1}, p_{2}\right)$ the corresponding momenta. $\mathbf{E}$ is a constant electric field and the "friction coefficient" $\zeta$ is chosen as a phase space function so that the kinetic energy (or speed) of the particle is conserved:

$$
\zeta=\mathbf{E} \cdot(\mathbf{p} / m) /\left(p^{2} / m\right) .
$$

Because of the conservation of kinetic energy, we may consider the reduced phase space at each value of the particle speed $v$, with coordinates $X=\left(q_{1}, q_{2}, \theta\right)$, where $\theta$ is the angle of the particle velocity vector with respect to the 1-direction. It is an elementrary calculation that

$$
\nabla_{X} \cdot \dot{X}=-\zeta
$$

so that the Liouville measure is not preserved when $\mathbf{E} \neq 0$. On the other hand, observe that Eqs. (1-2) define a flow $\left\{S_{\mathbf{E}}^{t}\right\}$ on the phase-space, running backward as well as forward, and that the inversion $\tilde{X}=\left(q_{1}, q_{2}, \theta-\pi\right)$, corresponding to velocity-reversal, has the property that $S_{\mathbf{E}}^{t}(\tilde{X})=\left(S_{\mathbf{E}}^{-t} X\right)^{\sim}$.

The model under consideration was previously studied theoretically and numerically in [30]. It is a simple example for a set of new methods in nonequilibrium molecular dynamics (NEMD) which has been developed in the past decade by W.G. Hoover, D.J. Evans, G.P. Morriss, S. Nosé and others [12, 20]. Unlike a more traditional approach (see, for example [28]) which models interactions of the physical system with a heat bath by including suitable stochastic elements in the dynamics, the new techniques are based upon dynamics which are purely deterministic and reversible but for which the Liouville theorem is invalid. In one version of the method, which we study, the total kinetic energy of a system of particles subjected to a theormodynamic or mechanical driving field is held fixed by modifying the dynamics according to a prescription of Gauss, the "principle of least constraint" [16]. What Gauss proposed was that forces $\mathbf{F}_{i}^{(c)}$ be added to the Newtonian dynamics in such a way that a chosen constraint $f(\mathbf{q}, \dot{\mathbf{q}}, t)=0$ be maintained and the total magnitude of the (Jacobi frame) constraint force,

$$
\sum_{i=1}^{N}\left(F_{i}^{(c)}\right)^{2} / m_{i},
$$

be minimized instantaneously. In the space of forces (or accelerations) the con straint defines a hyperplane by a linear equation $\sum_{i=1}^{N} \mathbf{n}_{i}(\mathbf{q}, \dot{\mathbf{q}}, t) \cdot a_{i}=b(\mathbf{q}, \dot{\mathbf{q}}, t)$, with $\mathbf{n}_{i}(\mathbf{q}, \dot{\mathbf{q}}, t)=\nabla_{\dot{\mathbf{q}}_{l}} f(\mathbf{q}, \dot{\mathbf{q}}, t)$. Since $\mathbf{F}_{\dot{i}}^{(c)} \propto \mathbf{n}_{i}$ by Gauss' principle, the final equations of motion are of the form:

$$
m_{i} \ddot{\mathbf{q}}_{i}=\mathbf{F}_{i}-\zeta \mathbf{n}_{i},
$$

for some $\zeta$. Obviously, our dynamics is a special case of this general construction. For a system of many particles, holding fixed the "peculiar" kinetic energy, $K=\frac{1}{2} \sum_{i=1}^{N} m_{i}\left(\dot{\mathbf{q}}_{i}-\mathbf{u}_{i}\right)^{2}\left(\mathbf{u}_{i}\right.$ is the expected velocity of particle $\left.i\right)$, should be equivalent to holding the temperature fixed, according to the identification 
$K=N \cdot d \cdot k_{B} T / 2$. In our simple example with $N=1$ the identification of "temperature" is not really appropriate, but we will use the term by analogy. In fact the analogy is best preserved, see [46], if we set $p^{2} / m=k_{B} T=\beta^{-1}$.

In practical simulations with realistic potentials and also in the simulations for our simple model in [30], the empirical measure

$$
\mu_{t}^{X} \equiv \frac{1}{t} \int_{0}^{t} d s \delta_{S^{s} X}
$$

appears to converge (weakly) to a final stationary distribution for at least a dense set of initial points $X$ of the phase space. However, the mathematical proof of existence and uniqueness of stationary measures, which can be given for some cases of the traditional stochastic approach $[17,18]$, is generally lacking here. Furthermore, in contrast to the stochastic modeling method, the stationary distributions appear on the basis of numerical evidence to be singular with respect to Liouville measure. Indeed, the measures appear to be multifractal, with an information dimension strictly less than the dimension of the constraint surface in phase space ([12], Ch. 10). The simple model we consider affords the opportunity to rigorously examine such issues.

In addition, simple formal arguments suggest some remarkable properties of the Gaussian dynamics. It is found in particular that the physical entropy production in the steady state is just equal to the asymptotic rate of decrease of the Gibbs entropy for time-evolved initial distributions, and the latter is seen to be just the negative of the sum of the Lyapunov exponents (defined almost surely with respect to the final stationary measure) [12]. From this results immediately a relation between the transport coefficients, which appear in the entropy production, and the Lyapunov exponents for the Gaussian dynamics. We wish also to study the validity of such relations in our simple model example.

The plan of the paper is as follows: In the next section of Part I we give simple formal arguments - which are later made into proofs - for the relations mentioned above, as well as indicate some generalizations and extensions which we do not prove afterward in all details. In the section following that, we give precise formulations of the rigorous results we establish for the model, and indicate some basic ideas of the argument. In addition, we point out the surprising transformation of this essentially non-equilibrium problem into a problem of (lattice) equilibrium Gibbs measures, by the method of Markov partitions and symbolic dynamics. In the final section of Part I we analyze in detail the basic features required of physical systems, in general, and of our model in particular for the validity of linear response theory. We do this in the context of the van Kampen argument against validity of linear response theory. In the more technical Part II the proofs of all the main results are outlined.

(b) Formal Arguments. Let us first give the argument for the relations between physical entropy production, time-derivative of Gibbs entropy, and Lyapunov exponents. Although our discussion is entirely in the context of the Lorentz model, it will appear that the basis of the results is rather general [12]. We assume in our discussion that, if $\mu$ is an initial measure absolutely continuous with respect to the Lebesgue measure and $\hat{S}_{\mathbf{E}}^{t} \mu=\mu \circ S_{\mathbf{E}}^{-t}$, then $\hat{S}_{\mathbf{E}}^{t} \mu \rightarrow \mu_{\mathbf{E}}^{t}$ (weakly) as $t \rightarrow+\infty$, where $\mu_{\mathbf{E}}^{+}$is the physical stationary measure for the forward evolution. For simplicity we denote $\hat{S}_{\mathbf{E}}^{t} \mu$ by $\mu_{t}$ and its density with respect to Liouville measure by $f_{t}$. With the 
usual definition of Gibbs entropy,

$$
S(\mu)=-k_{B} \int f(X) \log f(X) d X,
$$

it is a simple calculation for differentiable $f$ that

$$
\frac{d}{d t} S\left(\mu_{t}\right)=k_{B} \int f_{t}(X)\left(\nabla_{X} \cdot \dot{X}\right) d X=-k_{B} \mu_{t}(\zeta)
$$

using in the last equality Eq. (4). Observing from Eq. (3) that $\zeta$ is a bounded, continuous function on the phase space, we can therefore infer that

$$
\lim _{t \rightarrow+\infty}-\frac{d}{d t} S\left(\mu_{t}\right)=k_{B} \mu_{\mathbf{E}}^{+}(\zeta)
$$

and, in fact, the right side is just equal to $\mu_{\mathbf{E}}^{+}(\mathbf{v}) \cdot \mathbf{E} / T$. This has an interesting physical interpretation: if we consider $\mathbf{J} \equiv \mu_{\mathbf{E}}^{+}(\mathbf{v})$ as the steady state electrical current, then it is just $\mathbf{J} \cdot \mathbf{E} / T$, which is the entropy production due to Ohmic dissipation [19]. (It may appear odd that the negative time-derivative of Gibbs entropy corresponds to entropy production. One should understand that the Gaussian dynamics are supposed to model the effect of reservoir elements on the particle system, for which the total system, reservior + particles, obeys the Liouville theorem. Hence, the decrease of particle entropy corresponds to the increase of reservoir entropy, and the latter represents the physical entropy production.)

For the other half of the relation, we note that, if there exist local stable and unstable subspaces in the tangent space to $\mathfrak{M}$ (for every point where the flow is smooth), then one may define local exponential rates of contraction, $\Lambda_{\mathbf{E}}^{s}(X)$, and expansion, $\Lambda_{\mathbf{E}}^{u}(X)$, along those one-dimensional subspaces. The third direction, along the flow, is neutral. Formally, these rates are defined by the limits, $\Lambda_{\mathbf{E}}^{\alpha}(X)=\lim _{t \rightarrow 0} \frac{1}{t} \log \left\|\frac{\partial S_{\mathbf{E}}^{t}(X)}{\partial X} \cdot u_{\alpha}\right\|$, where $\frac{\partial S_{\mathbf{E}}^{t}(X)}{\partial X}$ is the Jacobian matrix of $S_{\mathbf{E}}^{t}$ at $X, \alpha=s(\alpha=u), u_{\alpha}$ is a vector in the stable (unstable) subspace at $x$, and $\|\cdot\|$ is the Euclidean norm. The volume of a small parallelepiped with one leg along the flow direction and the other legs along the stable and unstable rays has the volume which is the triple wedge product of those legs, i.e. the product of their magnitudes and a combination of trigonometric functions of the angles between the legs. We denote the latter angular factor by $U_{\mathbf{E}}(X)$, defined for all points of smoothness of the flow. Then, there holds the following relation for all $X$ at which the flow is not singular,

$$
\left(\nabla_{X} \cdot \dot{X}\right)(X)=\Lambda_{\mathbf{E}}^{s}(X)+\Lambda_{\mathbf{E}}^{u}(X)+\left.\frac{d}{d t} U_{\mathbf{E}}\left(S_{\mathbf{E}}^{t} X\right)\right|_{t=0} .
$$

On the other hand, $\lambda_{\mathbf{E}}^{s}=\mu_{\mathbf{E}}^{+}\left(\Lambda_{\mathbf{E}}^{s}\right)$ and $\lambda_{\mathrm{E}}^{u}=\mu_{\mathbf{E}}^{+}\left(\Lambda_{\mathbf{E}}^{u}\right)$ are the Lyapunov exponents for the ergodic measure $\mu_{\mathbf{E}}^{+}$, while the time-derivative has zero expectation just by stationarity under $S_{\mathbf{E}}^{t}$. Hence, we have also the relation

$$
\frac{1}{T} \mathbf{E} \cdot \mathbf{J}=-k_{B}\left(\lambda_{\mathbf{E}}^{s}+\lambda_{\mathbf{E}}^{u}\right)
$$

The content of this remarkable relation is the equality of the full (nonlinear) entropy production and the negative sum of the Lyapunov exponents. 
We now turn to a discussion of the formal response theory. It is very useful here to develop certain exact integral expressions for the stationary measures $\mu_{\mathbf{E}}^{+}$. To this end, let us note that the density of the measure at time $t$ starting from initial Lebesgue measure is just given by the Jacobian determinant

$$
f_{t}(X)=\left|\frac{\partial S_{\mathrm{E}}^{-t}(X)}{\partial X}\right| .
$$

Then, by an elementary calculation,

$$
\partial_{t} f_{t}(X)=-\left.\left(\nabla_{X} \cdot \dot{X}\right)\right|_{S_{\mathbf{E}}^{-t} X} \cdot f_{t}(X)=\beta\left(\mathbf{E} \cdot \mathbf{v}\left(S_{\mathbf{E}}^{-t} X\right)\right) \cdot f_{t}(X),
$$

and, by direct integration,

$$
f_{t}(X)=1+\beta \mathbf{E} \cdot \int_{0}^{t} \mathbf{v}\left(S_{\mathbf{E}}^{-s} X\right) f_{s}(X) d s=\exp \left[\beta \mathbf{E} \cdot \delta Q_{t}(X)\right] .
$$

Therefore, for any well-behaved function $\phi$ on the phase space,

$$
\mu_{t}(\phi)=\hat{S}_{\mathbf{E}}^{t} \mu_{0}(\phi)=\mu_{0}(\phi)+\beta \mathbf{E} \cdot \int_{0}^{t} \mu_{0}\left(\mathbf{v} \cdot\left(\phi \circ S_{\mathbf{E}}^{s}\right)\right) d s
$$

(where, note, $\mu_{0}$ according to our previous notation is just Lebesgue measure, which is stationary for $\mathbf{E}=0$.) By our assumption, $\hat{S}_{\mathbf{E}}^{t} \mu_{0}$ converges to $\mu_{\mathbf{E}}^{+}$as $t \rightarrow+\infty$, and, if the integrand on the right side has sufficiently good decay, we obtain finally

$$
\mu_{\mathbf{E}}^{+}(\phi)=\mu_{0}(\phi)+\beta \mathbf{E} \cdot \int_{0}^{\infty} \mu_{0}\left(\mathbf{v} \cdot\left(\phi \circ S_{\mathbf{E}}^{t}\right)\right) d t .
$$

Such exact expressions for steady-state measures are sometimes called in the physics literature "non-equilibrium statistical distributions" and have been known for a long time $[27,29,44]$. Assuming that $\mathbf{v}$ is in the class of $\phi$ for which the expression is valid, one obtains at once a formula for the exact current-response as a nonlinear function of field:

$$
\mathbf{J}(\mathbf{E})=\beta \mathbf{E} \cdot \int_{0}^{\infty} \mu_{0}\left(\mathbf{v} \otimes\left(\mathbf{v} \circ S_{\mathbf{E}}^{t}\right)\right) d t .
$$

This is an example of the so-called Kawasaki formula for the nonlinear response [41]. As we see below, it may be regarded as a generalization of the usual Green-Kubo formula.

It is now easy to specialize the above results to obtain the response to linear order in the field. Indeed, we see formally that the linear correction term to the measure $\mu_{\mathbf{E}}^{+}$is just given by

$$
\mu_{\mathbf{E}}^{+}(\phi)=\mu_{0}(\phi)+\beta \mathbf{E} \cdot \int_{0}^{\infty} \mu_{0}\left(\mathbf{v} \cdot\left(\phi \circ S_{0}^{t}\right)\right) d t+o(|\mathbf{E}|) .
$$

This is rigorously correct, for example, if there is a bound on the decay in $t$ of $\mu_{0}\left(\mathbf{v} \cdot\left(\phi \circ S_{\mathbf{E}}^{t}\right)\right)$ uniform in $\mathbf{E}$, so that dominated convergence may be applied to show the remainder term is really $o(|\mathbf{E}|)$. Needless to say, this is not just a fine point of rigor but is exactly where dynamical properties enter in the derivation of the transport law. In fact, from Eq. (10) one obtains directly Ohm's law

$$
\mathbf{J}=\boldsymbol{\sigma} \cdot \mathbf{E}+o(|\mathbf{E}|)
$$


with

$$
\boldsymbol{\sigma}=\beta \int_{0}^{\infty} \mu_{0}\left(\left(\mathbf{v} \circ S_{0}^{t}\right) \otimes \mathbf{v}\right) d t .
$$

The latter yields immediately the Einstein relation

$$
\boldsymbol{\sigma}=\beta \cdot \mathbf{D}
$$

where $\mathbf{D}$ is given by the usual Green-Kubo formula. Furthermore, putting together Eqs. (5), (11), and (12), we obtain a simple formula for the diffusion coefficient as:

$$
\hat{\mathbf{e}} \cdot \mathbf{D} \cdot \hat{\mathbf{e}}=\lim _{\mathbf{E} \rightarrow 0} \frac{-k_{B} T^{2}\left(\lambda_{\mathbf{E}}^{s}+\lambda_{\mathbf{E}}^{u}\right)}{\mathbf{E}^{2}},
$$

where $\hat{\mathbf{e}}$ is the unit vector $\mathbf{E} /|\mathbf{E}|$. The relation appears naturally as both the transport coefficient and Lyapunov exponents are related to entropy production.

There is nothing in the above arguments which imposes a restriction to $d=2$, and corresponding results for higher dimensions can be rigorously obtained by using extensions of our methods developed in [9]. Another interesting generalization is to consider the addition of an external magnetic field to the dynamics. This involves just the addition of an appropriate Lorentz force to the left side of Eq. (2):

$$
\dot{\mathbf{p}}=\mathbf{E}+\mathbf{p} \times \mathbf{B}-\zeta \cdot \mathbf{p} .
$$

Because the magnetic interaction is Hamiltonian and conserves kinetic energy, the definition of $\zeta$ is the same and also the formula (4) for the divergence of the dynamical vector-field remains valid. It is therefore easy to derive formally in the same manner as before expressions like Eqs. (8) and (9), with only $S_{\mathbf{E}}^{t}$ replaced by $S_{\mathbf{B}, \mathbf{E}}^{t}$. In particular, the Kawasaki-type formula for current response remains valid:

$$
\mathbf{J}(\mathbf{B}, \mathbf{E})=\beta \mathbf{E} \cdot \int_{0}^{\infty} \mu_{0}\left(\mathbf{v} \otimes\left(\mathbf{v} \circ S_{\mathbf{B}, \mathbf{E}}^{t}\right)\right) d t .
$$

The above formula describes several new phenomena that arise in the simultaneous presence of electric and magnetic fields, e.g. the Hall effect of transverse electrical currents. From this follows also the validity of Ohm's law, as in Eq. (11), but with a B-dependent conductivity given by

$$
\sigma(\mathbf{B})=\beta \int_{0}^{\infty} \mu_{0}\left(\left(\mathbf{v} \circ S_{\mathbf{B}, 0}^{t}\right) \otimes \mathbf{v}\right) d t .
$$

Although time-reversal symmetry is broken by the magnetic field, one can derive from this expression, by considering time-inversion, the relation

$$
\boldsymbol{\sigma}^{\top}(-\mathbf{B})=\boldsymbol{\sigma}(\mathbf{B})
$$

which is the usual Onsager-Casimir reciprocal relation for the transport coefficient.

(c) Rigorous Results. We now state precisely the results along the above lines that we can establish by - we emphasize - essentially just supplying necessary rigor to the above formal arguments. The basic properties we need, as we have seen, are existence of local stable and unstable manifolds, (weak) convergence to a stationary, ergodic measure and some uniform decay of correlations. The general approach to the rigorous derivation of the transport law is the same as that given in 
[13] for the case of stochastic lattice gases. Here the necessary information on uniform correlation bounds is established by approximating the deterministic dynamics by a suitable Markov chain, through the method of Markov sieves. Unfortunately, the formal argument as we have given it above cannot be presently made rigorous, since there are so far no proofs of decay of correlations for the Lorentz gas (even at zero field) in "true" time. Instead, such bounds have been obtained by counting time in terms of numbers of successive collisions. This is connected with the so-called special representation of the flow, a particular application of a general technique of ergodic theory. We give now a more precise formulation of our model than in the Introduction (partly to set notations) and then describe the special representation. Afterward, we formulate our main results as a series of propositions with brief indications of the main ideas of the proofs and, finally, discuss the notions of Markov partitions and symbolic dynamics which are not actually used in the proofs but give some additional insight into the model.

As stated in the Introduction, we consider the system of a moving particle in the torus $\mathbb{T}^{2}$ with a finite number of disjoint strictly convex scatterers. The region of that torus complementary to the union of all the scatterers is denoted by $Q$. Recall that the speed of the moving particle is constant and equals $v=p / m$. Therefore, the phase space of the system is now $\mathfrak{M}=Q \times S_{v}^{1}$, where $S_{v}^{1}$ stands for the circle of radius $v$. The motion under the field $\mathbf{E}$ generates the phase flow $\left\{S_{\mathbf{E}}^{t}\right\}$ in $\mathfrak{M}$ where $-\infty<t<\infty$. Define a subset of $\mathfrak{M}$ corresponding to the points of collision of the particle with the boundary $\partial Q$ :

$$
M=\left\{x=(\mathbf{q}, \mathbf{v}) \in \mathfrak{M}: \mathbf{q} \in \partial Q, \mathbf{v} \in S_{v}^{1}, \mathbf{v} \cdot \mathbf{n}(\mathbf{q}) \geqq 0\right\} .
$$

Here and further on $\mathbf{n}(\mathbf{q})$ stands for the inward unit normal vector to $\partial Q$ at the point $q$; we have chosen rather arbitrarily to label points by their velocity the instant after collision. Standard coordinates in $M$ are $r$ and $\varphi[4,5]$, where $r$ is the arc length parameter along $\partial Q$ and $\varphi$ is the angle between $n(q)$ and $v$ at a point $x=(q, v) \in M,|\varphi| \leqq \pi / 2$. Rather than $\varphi$ it is sometimes useful to consider the coordinate $s=\sin \varphi,|s| \leqq 1$. A map $T_{\mathrm{E}}: M \rightarrow M$ may be defined by taking each point $x \in M$ to the point of its next collision, the so-called billiard ball map or first-return map. We denote the time until the next collision by $\tau_{\mathbf{E}}(x)$ and note the important restriction of all our considerations to the case of finite horizon. In that case $\tau_{E}(x) \leqq \tau_{\max }<+\infty$ except possibly on the codimension 1 singularity set $S_{-1}=T_{\mathbf{E}}^{-1}(\partial M)$ of the piecewise smooth map $T_{\mathbf{E}}$ (where $\partial M=\{x=(\mathbf{q}, \mathbf{v}) \epsilon$ $M: \mathbf{v} \cdot \mathbf{n}(\mathbf{q})=0\}$.$) However, the singularities of T_{\mathbf{E}}$ are mild - they cannot accumulate, i.e. after a finite number of collisions the trajectory will escape the vicinity of $S_{-1}$ and stay uniformly far from it. The map $T_{\mathbf{E}}$ has an inverse $T_{\mathbf{E}}^{-1}$ which is also piecewise smooth with a singularity set $S_{1}=T_{\mathbf{E}}(\partial M)$. On the space $M$ there is defined the inversion $\tilde{x}=(r,-\phi)$ for $x=(r, \phi)$ under which $T_{\mathbf{E}}(\tilde{x})=\left(T_{\mathbf{E}}^{-1} x\right)^{\text {. }}$. Note that the billiard flow $\left\{S_{0}^{t}\right\}$ preserves the Liouville measure $d \mu_{0}=d \mathbf{q} d \mathbf{v}$ and the billiard map $T_{0}$ preserves the measure $d v_{0}=d r d s=\cos \varphi d r d \varphi$.

Since the dynamics for any $\mathbf{E}$ is completely deterministic, it is obvious that any point $(\mathbf{q}, \mathbf{v}) \in \mathfrak{M}$ is completely specified by giving the point $x \in M$ corresponding to its state just before its last collision and the time $\tau$ since that last collision. This is the so-called special representation of the flow. More formally, it corresponds to representing the system $\left(\mathfrak{M}, S_{\mathbf{E}}^{t}\right)$ as the flow under the (ceiling) function $\tau_{\mathbf{E}}$ generated by the map $T_{\mathrm{E}}$ on the (base) space $M$; e.g. see the general discussion in Chapter 11 of [10]. Let us just remark here that if $v_{\mathbf{E}}$ is a measure on $M$ invariant (ergodic) 
under $T_{\mathbf{E}}$, then a measure $\mu_{\mathbf{E}}$ on $\mathfrak{M}$ invariant (ergodic) under $S_{\mathbf{E}}^{t}$ is defined by

$$
\mu_{\mathbf{E}}(f)=\frac{1}{\bar{\tau}_{\mathbf{E}}} \int_{M} v_{\mathbf{E}}(d x) \int_{0}^{\tau_{\mathbf{E}}(x)} d \tau f(x, \tau)
$$

for $f \in C(\mathfrak{M})$, using the isomorphism $\mathfrak{M} \cong\left\{(x, \tau): x \in M, 0 \leqq \tau \leqq \tau_{\mathbf{E}}(x)\right\}$. Here, $\bar{\tau}_{\mathbf{E}} \equiv v_{\mathbf{E}}^{+}\left(\tau_{\mathbf{E}}\right)$, the mean collision time, appears as a denominator for proper normalization. Notice that the measures $\mu_{0}, v_{0}$ previously defined are in fact so related. There is also a simple relation between the Lyapunov exponents $\tilde{\lambda}_{\mathbf{E}}^{u, s}$ for any ergodic measure $\nu_{\mathbf{E}}$ under $T_{\mathbf{E}}$ and those for the associated $\mu_{\mathbf{E}}$, which is just $\lambda_{\mathbf{E}}^{u, s}=\tilde{\lambda}_{\mathbf{E}}^{u, s} / \bar{\tau}_{\mathbf{E}}$.

We can now state our results. They all require the condition of finite horizon and sufficient smallness of the field, which we hereafter assume without explicit mention. First, we have (defining, as above, $\hat{T}_{\mathbf{E}}^{n} v \equiv v \circ T_{\mathbf{E}}^{-n}$ )

Proposition 1. There is a stationary, ergodic measure $\nu_{\mathbf{E}}^{+}$for the map $T_{\mathbf{E}}$ on $M$, which is the weak limit

$$
v_{\mathbf{E}}^{+}=w-\lim _{n \rightarrow+\infty} \hat{T}_{\mathbf{E}}^{n} v,
$$

for any measure $v \ll v_{0}$ on $M$. The measure $v_{\mathbf{E}}^{+}$satisfies the Pesin formula for the K-S entropy

$$
h_{v_{\mathbf{E}}^{+}}\left(T_{\mathbf{E}}\right)=\tilde{\lambda}_{\mathbf{E}}^{u},
$$

and Young's formula for the fractal (or information) dimension

$$
H D\left(v_{\mathbf{E}}^{+}\right)=h_{v_{\mathbf{E}}^{+}}\left(T_{\mathbf{E}}\right)\left(\frac{1}{\tilde{\lambda}_{\mathbf{E}}^{u}}-\frac{1}{\tilde{\lambda}_{\mathbf{E}}^{s}}\right) .
$$

Furthermore, considering $S_{\mathrm{E}}^{t}$ as the special flow, one obtains by the construction in Eq. (14) above, an invariant ergodic measure $\mu_{\mathbf{E}}^{+}$which is the weak limit $\mu_{\mathbf{E}}^{+}=w-\lim _{t \rightarrow+\infty} S_{\mathbf{E}}^{t} \mu$ for any $\mu \ll \mu_{0}$, which has the K-S entropy given by $h_{\mu_{\mathbf{E}}^{+}}\left(S_{\mathbf{E}}^{1}\right)=h_{v_{\mathbf{E}}^{+}}\left(T_{\mathbf{E}}\right) / \bar{\tau}_{\mathbf{E}}$ and the fractal dimension $H D\left(\mu_{\mathbf{E}}^{+}\right)=H D\left(v_{\mathbf{E}}^{+}\right)+1$.

This result is proved in Part II, Sects. (d) and (f). It is easy to check by combining the Pesin and Young formulas that

$$
H D\left(\mu_{\mathbf{E}}^{+}\right)=2+\left(1+\frac{\overline{\zeta_{\mathbf{E}}}}{h_{\mathbf{E}}}\right)^{-1},
$$

where $h_{\mathbf{E}} \equiv h_{\mu_{\mathbf{E}}^{+}}\left(S_{\mathbf{E}}^{1}\right)$ and $\overline{\zeta_{\mathbf{E}}} \equiv-\left(\lambda_{\mathbf{E}}^{u}+\lambda_{\mathbf{E}}^{s}\right)$. The next main result allows us to physically identify the latter expression:

Proposition 2. For any measure $\mu$ on $\mathfrak{M}, \mu \ll \mu_{0}$,

$$
\mathbf{J} \cdot \mathbf{E} / T=-\lim _{t \rightarrow+\infty} \frac{d}{d t} S\left(\mu_{t}\right)=-k_{B}\left(\lambda_{\mathbf{E}}^{u}+\lambda_{\mathbf{E}}^{s}\right) .
$$

Here, as above, $\mathbf{J}=\mu_{\mathbf{E}}^{+}(\mathbf{v}), \mu_{t}=\hat{S}_{\mathbf{E}}^{t} \mu$ and $\lambda_{\mathbf{E}}^{u}, \lambda_{\mathbf{E}}^{s}$ are Lyapunov exponents for the ergodic measure $\mu_{\mathbf{E}}^{+}$.

This result follows from the weak convergence in Proposition 1 and the existence of stable and unstable subspaces in $\mathfrak{I}_{X} \mathfrak{M}$ except for the codimension 1 set of points $X \in \mathfrak{M}$ where the flow is singular. The existence of the subspaces is 
guaranteed by the strong hyperbolicity of the flow $S_{\mathbf{E}}^{t}$, which holds for small $\mathbf{E}$ (see Part III and Ref. [23]). It is perhaps interesting to note that the ratio determining the fractal dimension is just the physical entropy production $\mathbf{J} \cdot \mathbf{E} / T$ divided by $\left(k_{B}\right.$ times) the dynamical entropy production $h_{\mathbf{E}}$.

To state the next results, we must introduce some additonal notation. For any $X \in \mathfrak{M}$ let us define $\mathbf{Q}(X)$ to be the projection onto the configuration space $Q$, i.e. $\mathbf{Q}(\mathbf{q}, \mathbf{v})=\mathbf{q}$. In particular, $\mathbf{Q}(x) \in \partial Q$ for $x \in M$. We then define on $M$ a function $\Delta_{\mathbf{E}}$ by

$$
\Delta_{\mathbf{E}} \equiv \mathbf{Q} \circ T_{\mathbf{E}}-\mathbf{Q}
$$

Clearly, $\Delta_{\mathbf{E}}(x)$ is just the total vector displacement of the particle from its starting point $x \in M$ until its next collision. This definition is actually ambiguous since we use periodic boundary conditions on $Q$. For the results below it is crucial that $\boldsymbol{\Delta}_{\mathbf{E}}$ be defined by the convention that, when a particle in $\mathbb{R}^{2}$ crosses the boundary of the fundamental domain $Q$, the displacement is evaluated between the starting point in $Q$ and the final position in the adjacement domain (rather than its periodic image in the fundamental domain). Let us also define a space of Hölder continuous functions $H_{\alpha}=\left\{f:|f(x)-f(y)| \leqq C_{f}|x-y|^{\alpha}\right.$ for any $\left.x, y \in M\right\}$. More generally, let $H_{\alpha}^{*}$ denote the space of piecewise Hölder continuous functions, which are Hölder continuous (with an exponent $\alpha$ ) on a finite collection of subdomains in $M$ separated by a finite union of compact smooth curves. (Note that the curves and domains must be fixed for the class $H_{\alpha}^{*}$ under consideration: we consider below the case where the discontinuities occur on the singularity sets $S_{-1,1}=S_{-1} \cup S_{1}$ of both the maps $T_{\mathbf{E}}^{ \pm}$and $T_{0}^{ \pm}$). Then, an exact integral formula for invariant expectations and a Kawasaki-type formula for nonlinear response can be established in the following form:

Proposition 3. For any $\phi \in H_{\alpha}^{*}$,

$$
v_{\mathbf{E}}^{+}(\phi)=v_{0}(\phi)+\sum_{n=1}^{\infty} v_{0}\left[\left(\phi \circ T_{\mathbf{E}}^{n}\right)\left(1-e^{-\beta \mathbf{E} \cdot \Delta_{\mathbf{E}}}\right)\right] .
$$

Furthermore, the following equality holds

$$
\mathbf{J}(\mathbf{E})=\frac{1}{2 \bar{\tau}_{\mathbf{E}}} v_{0}\left[\Delta_{\mathbf{E}}\left(1-e^{-\beta \mathbf{E} \cdot \Delta_{\mathbf{E}}}\right)\right]+\frac{1}{\bar{\tau}_{\mathbf{E}}} \sum_{n=1}^{\infty} v_{0}\left[\left(\Delta_{\mathbf{E}} \circ T_{\mathbf{E}}^{n}\right)\left(1-e^{-\beta \mathbf{E} \cdot \Delta_{\mathbf{E}}}\right)\right] .
$$

The proof of this proposition is obtained by repeating the formal arguments given earlier, but now for the map $T_{\mathbf{E}}$ (see Part II(a).) To make the argument rigorous it must be shown, for the first Eq. (16), that the function $f_{\mathbf{E}}(x)=1-e^{-\beta \mathbf{E} \cdot \Delta_{\mathbf{E}}(x)}$ obeys $v_{0}\left(f_{\mathbf{E}}\right)=0$ and is in $H_{\alpha}^{*}$ for all (small $\mathbf{E}$ : the result then follows from Theorem 17 of Part II(e) which proves the decay of correlations to guarantee convergence of the summation. The second equation follows more or less directly from the first one (see II(a)).

Finally, we have the following results on the linear response, transport law and Einstein relation:

Proposition 4. For any $\phi \in H_{\alpha}^{*}$,

$$
v_{\mathbf{E}}^{+}(\phi)=v_{0}(\phi)+\beta \mathbf{E} \cdot \sum_{n=1}^{\infty} v_{0}\left[\left(\phi \circ T_{0}^{n}\right) \boldsymbol{\Delta}_{0}\right]+o(|\mathbf{E}|) .
$$


Furthermore, $\mathbf{J}=\boldsymbol{\sigma} \cdot \mathbf{E}+o(|\mathbf{E}|)$, where $\boldsymbol{\sigma}=\beta \mathbf{D}$ and

$$
\mathbf{D}=\frac{1}{2 \bar{\tau}_{0}} \sum_{n=-\infty}^{+\infty} v_{0}\left(\left(\boldsymbol{\Delta}_{0} \circ T_{0}^{n}\right) \otimes \boldsymbol{\Delta}_{0}\right) .
$$

The latter expression is just the (discrete-time) Green-Kubo formula established by Sinai and Bunimovich in [3] for the diffusion coefficient. See also [5], where this formula was corrected and the matrix $\mathbf{D}$ was shown to be positivedefinite. In these works, the diffusion coefficient is naturally defined through the covariance of the limiting Wiener process for the rescaled particle motion in the Lorentz array of scatterers. Thus, the relation $\sigma=\beta \mathbf{D}$ is a proper form of the Einstein relation. (Although we give no details here, similar results can be obtained for the situations with an external magnetic field, as long as the scatterer array has finite-horizon for the motion along circular arcs produced by the Lorentz force. The limiting Brownian motion with B-dependent drift can be obtained, for example, by the arguments in [9], and the diffusion coefficient is then given just by the above Green-Kubo formula with $T_{0}^{n}$ replaced by $T_{\mathbf{B}, 0}^{n}$. In that case, therefore, there is a generalized Einstein relation of the form $\tilde{\boldsymbol{\sigma}}(\mathbf{B})=\beta \mathbf{D}(\mathbf{B})$, where $\tilde{\boldsymbol{\sigma}}$ is the symmetric part of $\boldsymbol{\sigma}$ given by the formula (13) or by a similar discrete-time formula.) The proof of Eq. (17) follows from the uniform correlation bounds in Part II(e), which allows dominated convergence to be applied to show the remainder term is $o(|\mathbf{E}|)$. The rest of the proposition then follows rather directly (see II(a).) From Ohm's law and Eq. (15), we can obtain an estimation for the fractal dimension valid for small $\mathbf{E}$ :

\section{Corollary.}

$$
H D\left(\mu_{\mathbf{E}}^{+}\right)=3-\frac{\mathbf{E}^{\top} \cdot \boldsymbol{\sigma} \cdot \mathbf{E} / T}{k_{B} \cdot h_{0}}+o\left(|\mathbf{E}|^{2}\right) .
$$

This requires the proof that $\lim _{\mathbf{E} \rightarrow 0} h_{\mathbf{E}}=h_{0}$ (see Part II(f).) In particular, it follows that $H D\left(\mu_{\mathbf{E}}^{+}\right)<3$ when $\mathbf{E}$ is small but finite.

The method of Markov sieves is the main technical tool used both in the construction of the invariant measure and in the proof of the correlation bounds. The construction of the measure proceeds by first defining in a usual manner a conditional measure $p^{c}$ on unstable fibers $\gamma^{u}(x)$ and, then, for a selected fiber $\gamma^{u}$, proving both the existence of the limit $w-\lim _{n \rightarrow+\infty} \hat{T}_{\mathrm{E}}^{n} p^{c}$ and its independence of the choice of $\gamma^{u}$. Without entering into any technical details, we just remark that $\hat{T}_{\mathbf{E}}^{n} p^{c}(B)$ - for a fixed "parallelogram" $B$ circumscribed by stable and unstable fibers - is shown to have a limit only by studying the simultaneous evolution of the whole collection of parallelograms composing the Markov sieve. Likewise, the correlation bounds are obtained by using the Markov sieve to approximate the entire evolution sufficiently well by a Markov chain with good decay of correlations. The uniformity in $\mathbf{E}$ is the consequence of a "stability" property of the strictly hyperbolic map $T_{0}$ under small perturbations.

The related method of Markov partitions, which played the crucial role in earlier work $[2,3]$, is not used here. Nevertheless, it should be possible to construct such partitions with good enough properties, and, not only would this yield some further conjectured results, but also some additional heuristic insights into the model. We therefore very briefly explain this method and the related idea of symbolic dynamics. A Markov partition is a certain countable partition $\eta$ of $M$ into 
parallelograms $\eta=\left\{A_{i}: i \in J\right\}$ (for the definition of parallelograms see our Sect. II(c). As a notation, we write $\gamma_{\eta}^{u}(x)=\gamma^{u}(x) \cap A_{i}$, where $x \in A_{i} \in \eta$ and $\gamma^{u}(x)$ is the local unstable fiber through $x$ (with a similar notation for the stable fibers.) Then, the Markov partition has the fundamental property that for a.e. $x \in M$,

$$
T\left(\gamma_{\eta}^{s}(x)\right) \subseteq \gamma_{\eta}^{s}(T x), \quad T^{-1}\left(\gamma_{\eta}^{u}(x)\right) \subseteq \gamma_{\eta}^{u}\left(T^{-1} x\right) .
$$

It can be shown that the sets of the form $\bigcap_{n=-\infty}^{+\infty} T^{n} A_{\omega_{n}}$ for any sequence $\omega=\left\{\ldots, \omega_{-n}, \ldots, \omega_{0}, \ldots, \omega_{n}, \ldots\right\} \in \mathfrak{T}^{\mathbb{Z}}$ may consist only of one or no points, and $\bigcap_{n=-\infty}^{+\infty} T^{n} A_{\omega_{n}} \neq \emptyset$ if $v\left(A_{\omega_{n}} \cap T A_{\omega_{n+1}}\right)>0$ for all $n \in \mathbb{Z}$. Setting $\pi_{i j}=1$ if $v\left(A_{i} \cap T A_{j}\right)>0$ and 0 otherwise, we may define the space $\Omega$ of symbolic sequences $\omega$ as the subset of $\mathfrak{I}^{\mathbb{Z}}$ such that $\pi_{\omega_{n} \omega_{n+1}}=1$ for every $n \in \mathbb{Z}$. Then it may be seen that the mapping $\Phi: \Omega \rightarrow M$ defined as

$$
\phi(\omega)=\bigcap_{n=-\infty}^{+\infty} T^{n} A_{\omega_{n}}
$$

is one-to-one onto a set of full $v$-measure. It a natural way it gives an isomorphism of measurable spaces for $M$ and $\Omega$. Furthermore, if $S$ is the shift on $\Omega$ defined by $(S \omega)_{n}=\omega_{n-1}$, then $\Phi \circ S=T \circ \Phi$ so that $v$ is pulled back to an $S$-invariant measure $\rho$ on $\Omega$. In fact, the essential point is that $\rho$ is a kind of one-dimensional lattice Gibbs measure for the spin-system with values of spin in the countable set $\mathfrak{J}$ and with interactions of sufficiently rapid spatial decay. The formal Hamiltonian of the measure $\rho_{\mathbf{E}}^{+}$(corresponding to $\nu_{\mathbf{E}}^{+}$) is just given by the expression

$$
H(\omega) \approx \sum_{n=-\infty}^{+\infty} \tilde{\Lambda}_{\mathbf{E}}^{u}\left(\Phi\left(S^{n} \omega\right)\right),
$$

in which quantity $\tilde{\Lambda}_{\mathbf{E}}^{u}(x)$ is the local exponential rate of expansion for $x \in M$ under the map $T_{\mathbf{E}}$, and is simply related to the expansion rate for $S_{\mathbf{E}}^{t}$ by $\tilde{\Lambda}_{\mathbf{E}}^{u}(x)=$ $\int_{0}^{\tau_{\mathbf{E}}(x)} d \tau \Lambda_{\mathbf{E}}^{u}(x, \tau)$. (For the notions, see Ref. [33].) What is very remarkable about this isomorphism in our context is that it converts the essentially nonequilibrium measure $v_{\mathbf{E}}^{+}$into a lattice Gibbs measure $\rho_{\mathbf{E}}^{+}$of an equilibrium spin-system! This is the main feature of the so-called thermodynamical formalism [33].

This transformation, if it can be rigorously carried out here, should have various consequences. First, it is believed on the basis of various numerical evidence that the measure $v_{\mathbf{E}}^{+}$should be multifractal, with an entire continuous spectrum of associated dimensions. In fact, we expect on general grounds that this should be so (for small $\mathbf{E}$ ) and the verification by the above method of symbolic representation should be possible along the lines in [39]. Another interesting consequence of the representation as a lattice Gibbs measure is that there should be a variational principle which characterizes the measure $v_{\mathbf{E}}^{+}$. Specifically, the measure $v_{\mathbf{E}}^{+}$should minimize the quantity $f(v) \equiv v\left(\widetilde{\Lambda}_{\mathbf{E}}^{u}\right)-h_{v}\left(T_{\mathbf{E}}\right)$ among all the ergodic measures for $T_{\mathrm{E}}$ on $M$. The question of existence of a variational characterization of steady state measures has been a traditional one in non-equilibrium statistical mechanics, with a principle of minimum entropy production most often proposed [13]. It may be of some interest to observe that linear corrections to invariant measures, such as in Eq. (17) above, are known to be correctly prescribed by minimizing entropy production in some simple stochastic dynamical contexts $[13,24]$. However, no such principle is known to be exactly valid, and the present example is the only one we know where a microscopic measure is precisely characterized. It may be that this example is, in fact, a little more reminiscent of the maximum entropy principle proposed by Zubarev [45]. On the other hand, it must 
be admitted that the form of the principle considered here is quite different from any of the ones conventionally considered in non-equilibrium statistical mechanics. The minimization is only within the class of ergodic measures and, while $f(v) \geqq 0$ in that class, the minimum should be exactly zero (so that $v_{\mathbf{E}}^{+}$satisfies the Pesin formula.) Whereas standard variational principles seek to characterize a (unique) invariant measure out of the class of all probability measures, here the principle characterizes a certain physical measure out of the infinite class of ergodic measures for the deterministic dynamics.

A final remark which we make regarding the Gibbsian formulation of our problem, is that, in this guise, the conductivity $\sigma$ appears as a "susceptibility," or the equilibrium response to an applied field. That is, the current $\mathbf{J}$ is just the expected value of a variable $\mathbf{A}_{\mathbf{E}}$ (actually, $\mathbf{A}_{\mathbf{E}}=\boldsymbol{\Delta}_{\mathbf{E}} / \bar{\tau}_{\mathbf{E}}$ : see Part II, (a)), and it is possible to show that the Green-Kubo formula for the conductivity $\left.\boldsymbol{\sigma} \equiv \nabla_{\mathbf{E}} \mathbf{J}\right|_{\mathbf{E}=0}$ is equivalent to

$$
\left.\nabla_{\mathbf{E}} v_{\mathbf{E}}^{+}\left(\mathbf{A}_{\mathbf{E}}\right)\right|_{\mathbf{E}=0}=v_{0}\left(\mathbf{A}_{0}\left(-\left.\nabla_{\mathbf{E}} H\right|_{\mathbf{E}=0}\right)\right)
$$

where $H$ is the formal Hamiltonian in Eq. (19). This is exactly the usual perturbation formula for the response of an equilibrium system to a small change in the potential.

(d) The van Kampen Argument Against Linear Response. In the original form given by Kubo [26], the linear response theory essentially amounted to deriving a formula like our Eq. (7) - for the equilibrium measure $\mu_{0}$ evolved to time $t$ - but without a thermostating mechanism. Thereafter, an expression was obtained for the current response by first expanding in $\mathbf{E}$ and then taking the limit $t \rightarrow+\infty$, in that order of limits. Because no "heat bath" was employed, the stationary state did not exist and the opposite order could not be taken. Although it is the opposite order which is logically correct, the final result for the linear part of the response was exactly the same as ours (essentially because the dissipative effects of the "heat bath" are $O\left(|\mathbf{E}|^{2}\right)$ and do not enter at the retained order). Alternately, one may simply expand the expectation in $\mathbf{E}$ at a finite macroscopic time, with the assumption that the resulting finite-time integral contains essentially the full contribution to the conductivity.

Kubo's analysis was criticized by van Kampen in [22]. He claimed that the mathematical condition necessary for validity of the derivation is a restriction on the field strength sufficient to guarantee closeness of an individual trajectory over this macroscopic time interval. The basic point of the van Kampen argument was that one cannot expect such a linear microscopic response (i.e. of individual particle trajectories) over macroscopic times like seconds, minutes, or hours under an external perturbation, unless that perturbation is exceedingly small. On this basis he challenged the theoretical foundation of Kubo's microscopic response theory derivations of macroscopic linear transport laws (including, perhaps, the predicted value of the phenomenological coefficient). To demonstrate his point, van Kampen considered electrical conduction in a system of electrons which move freely except for occasional collisions with impurities. An external field $\mathbf{E}$ then has the effect of displacing the particle paths from their unperturbed positions over a time $t$ by $a t$ least an amount $\frac{1}{2} t^{2}(e|\mathbf{E}| / m)$. van Kampen argued that, in order that the induced current be linear in $|\mathbf{E}|$, one must have $\frac{1}{2} t^{2}(e|\mathbf{E}| / m) \ll d$, where $d$ is a mean spacing of impurities. Obviously, the upper bound on allowed field strengths $|\mathbf{E}|$ becomes 
more severe as $t$ increases. Taking $d \approx 100 \AA$ and $t$ to be a macroscopic time of order 1 second or so, van Kampen found that the field must be less than $\approx 10^{-18} \mathrm{~V} / \mathrm{cm}$ !

We argue that, in fact, much weaker conditions are sufficient for validity of linear response than such a severe requirement of "microscopic linearity": it is certainly nowhere used in our proof. We believe the loophole in van Kampen's argument comes from the fact that linear response theory deals actually with probability distributions of the electrons rather than individual phase space points. Such distributions of electrons are the relevant objects when one deals with a macroscopic system containing many particles. We agree therefore with the discussion of Kubo et al. in Sect. 4.7.2 of [37]. If one examines the formal response theory calculations, for example which lead to our Eq. (9) in Sect. (b), it is apparent that it really requires only relatively modest assumptions. The main requirement is that of a uniform bound on decay of correlations in the Green-Kubo integrand. The observation made in [12], Sect. 7.8, that the integrands in formulas like Eq. (10) typically decay to nearly zero in a microscopic time, makes it very reasonable that such uniform integrability as is required will hold. Therefore, the invariant measure of realistic systems have very likely a finite, linear correction such as in Eq. (9). Furthermore, for such a system the macroscopic current response will be the sum of separate contributions of many individual electrons. Therefore, one should expect that there will be a law of large numbers for the macroscopic current, so that for every phase space point distributed with respect to the invariant measure the actual, empirical value of the current is equal to the average value with a probability approaching unity as the number of electrons increases (e.g. see [14]). Since the average value has a perfectly linear behavior in $|\mathbf{E}|$, so will the actually observed current response.

The substantive issue raised by van Kampen's criticism is just what is the exact range of validity of the response theory. One part of the question is what range of $\mathbf{E}$ constitutes the linear regime, where the linear transport law is valid, and whether the observed conductivity in the macroscopic linear regime corresponds to Kubo's prediction. It is possible in principle that what is experimentally observed corresponds to an intermediate asymptotic regime, where nonlinear corrections conspire to produce a linear effect with modified slope (although this seems unlikely). Another question is what is the range of $\mathbf{E}$ for which the full nonlinear response formula, like Eq. (9), is valid. We address now the latter issue in the context of our concrete model.

Consideration of the details in Part II yields conditions on $\mathbf{E}$ of a similar form as van Kampen's but with a crucial difference. A main condition of our proof is that of strict hyperbolicity, and that requires that the dynamics with imposed field be a sufficiently small perturbation of the original free billiards. A condition on $\mathbf{E}$ sufficient for that purpose is that $\frac{1}{2} \bar{\tau}^{2}(e|\mathbf{E}| / m) \ll a$, where $\bar{\tau}$ is the mean-free-time between collisions and $a$ is the radius of the scatterers. The meaning of this condition is that, in the time it takes until its next collision, a particle should deviate from its original trajectory under an applied field by an amount which is a small fraction of the scatterer radius. This guarantees that the collision map $T_{\mathbf{E}}$ with an external field is a small perturbation of the original discrete dynamics $T_{0}$. Here, the demand of finite horizon requires that $a$ be only a little smaller than the distance $d$ between scatterers. Therefore, the main difference between this estimate and van Kampen's is the appearance of the microscopic mean-free-time - which is also of the order of the relaxation or correlation-decay time - rather than a macroscopic time 
of the order of seconds. Some similar but more stringent requirements emerge from the specific details of the proof in Part II. For example, in Lemmas 10 and 11 it is assumed that the perturbation is small under $m$ iterates of the map $T_{\mathbf{E}}$, where $m$ is an integer selected so that the minimal expansion rate after $m$ collisions, $W^{m}$, should be greater than, say, $100 m$. This requires $m=30$ or so, and the basic restriction on $\mathbf{E}$ is of the form $\frac{1}{2} \bar{\tau}^{2}(e|\mathbf{E}| / m) W^{30} \ll d$ (cf. the discussion in [31]). The expansion factor $W \approx d / a$ appears since deviations accumulate exponentially; it may be taken somewhat less than 2 . If one estimates the mean free time by taking a scatterer separation $d \approx 100 \AA$ and $v \approx 10^{8} \mathrm{~cm} / \mathrm{sec}$, a typical Fermi velocity, then $\bar{\tau}=d / v \approx 10^{-14} \mathrm{sec}$. Therefore, requiring that the deviation in 30 collisions be, say, no more than $\frac{1}{100}$ of a scatterer radius, we still arrive at an estimate for $\mathbf{E}$ which is $10^{18}$ times bigger than van Kampen's, allowing electric fields up to at least about $1 \mathrm{~V} / \mathrm{cm}$.

Unfortunately, for the proof in its present form, a much more severe restriction comes from Lemma 13, which makes a similar requirement on closeness of a power of the collision-map but with the number of iterates $n_{2}$ selected by the requirement of "sufficient mixing" in the phase space. It is more difficult to estimate the minimum size of $n_{2}$, but a value of 1000 could probably suffice. Even though this is still a microscopic time, it already implies an extremely tiny bound on allowed $\mathbf{E}$, because of the huge factor $W^{1000}$. The resulting bound is much smaller than van Kampen's! Actually, van Kampen's numerical estimate did not take properly into account exponential deviation of trajectories - as he himself remarked, it is "far too optimistic." The true requirement on $\mathbf{E}$ for microscopic linear response over macroscopic times is smaller than his stated estimate by a factor of $W^{10^{14}}$ ! That is the necessary limit on $\mathbf{E}$ he could have claimed, if his restrictive condition were really required, and our estimate is much better than that. However, our proof as it stands certainly does not prove the transport law for a realistic range of $\mathbf{E}$.

We have two comments on the situation:

First, the strong requirement in Lemma 13 might be eliminated with some greater effort to optimize its proof. The essential requirement of our strategy is, we believe, a closeness of the trajectory for just several collisions and that implies a reasonable range of validity of the response theory. We emphasize again that our proof nowhere uses any "linearity" of individual, microscopic trajectories over a macroscopic time interval. Such a closeness is required only over a fixed, microscopic time interval. The consequence of the closeness of the single collision map is a certain "stability" of the phase-portrait of the dynamics as a whole. The entire hyperbolic structure of the billiards at zero field - local stable and unstable manifolds, Markov partitions, etc. - are just "slightly distorted" by turning on the thermostat and small field. Since we are only interested ultimately in statistical information about our system, it is this stability of the whole dynamics which is relevant and not the stability of individual trajectories over macroscopic times. From such properties we can deduce, in particular, the uniform estimate on correlation decay.

Second, for the derivation of the transport law it is just this uniform integrability, besides existence of the stationary measure itself, which are required. The specific conditions on $\mathbf{E}$ emerge from our particular strategy based on strict hyperbolicity to prove a priori these requirements. There is no question that the required integrability is more general than the strict hyperbolicity, which can be lost if even a single scattering surface has a point of zero curvature. One must carefully distinguish between the linear response argument itself, which should 
have very general correctness, and the specific technique we have used here to establish the required integrable decay.

\section{Mathematical Proofs}

Here we supply the proofs of all the main results in Part I. First, we repeat in Sect. (a) the formal response theory calculations in terms of the billiard map $T_{\mathbf{E}}$, but taking care to point out what is needed to make the argument into a proof. Afterward, we turn to the more difficult problem of existence of the limit measure $v_{\mathbf{E}}^{+}$and investigation of its ergodic and statistical properties. Our reasonings here are very much similar to those employed in the theory of hyperbolic billiards [2-6, $25,35]$. Recently this theory has been extended also to certain billiard-like Hamiltonian systems $[11,7]$ and to piecewise linear hyperbolic maps of the torus $[38,8]$. This theory is now sufficiently far developed, so that we can only outline here the corresponding arguments. We will explain in detail only the properties of our model which differ from those of billiards and other related systems. The main difference is certainly the absence of an absolutely continuous invariant measure in the phase space. In Sect. (b) the existence of local stable and unstable fibers for $T_{\mathbf{E}}$ in $M$ (and for $S_{\mathrm{E}}^{t}$ in $\mathfrak{M}$ ) is established for almost every point with respect to the zero-field measures. In Sect. (c) the main tools for study of the statistical properties investigated. In the longest Sect. (c) the main tools for study of the statistical properties of the model, the so-called homogeneous fibers and the Markov sieve, are introduced and investigated. In the longest Sect. (d) the stationary measure $v_{\mathbf{E}}^{+}$is constructed and some basic probability estimates developed. In Sect. (e) the uniform estimates on decay of correlations are established which are needed to prove the response formulas and Einstein relation. Finally, in Sect. (f) the Pesin and Young formulas are established, and their limiting behavior for small $\mathbf{E}$ is investigated as well.

The smallness of the field $\mathbf{E}$ is always assumed as well as the condition of finite horizon. Throughout the text we denote by $c_{1}, c_{2}, \ldots$ various positive constants (usually, constant factors), by $a_{1}, a_{2}, \ldots$ also positive constants (usually, exponents) and by $\alpha_{1}, \alpha_{2}, \ldots$ various positive numbers which are less than 1 .

(a) Response Calculation for the Discrete-Time Map. We first make the calculation for the formula in Eq. (16) of Proposition 3 which gives the expectations with respect to $v_{\mathbf{E}}^{+}$. Obviously, for any function $\phi$ on $M$,

$$
\begin{aligned}
\hat{T}_{\mathbf{E}}^{n} v_{0}(\phi) & =v_{0}(\phi)+\sum_{k=1}^{n}\left[v_{0}\left(\phi \circ T_{\mathbf{E}}^{k}\right)-v_{0}\left(\phi \circ T_{\mathbf{E}}^{k-1}\right)\right] \\
& =v_{0}(\phi)+\sum_{k=1}^{n} v_{0}\left[\left(\phi \circ T_{\mathbf{E}}^{k}\right)\left(1-\frac{d\left(\dot{T}_{\mathbf{E}}^{-1} v_{0}\right)}{d v_{0}}\right)\right] .
\end{aligned}
$$

To evaluate the Radon-Nikodým derivative $d\left(\hat{T}_{\mathbf{E}}^{-1} v_{0}\right) / d v_{0}$, we recall first the obvious fact that

$$
\frac{d\left(\hat{S}_{\mathbf{E}}^{t} \mu_{0}\right)}{d \mu_{0}}=\left|\frac{\partial S_{\mathbf{E}}^{-1}(X)}{\partial X}\right|
$$


The Jacobian determinant obeys the Eq. (6) in Part I which may be explicitly integrated to give

$$
\left|\frac{\partial S_{\mathbf{E}}^{-t}(X)}{\partial X}\right|=\exp \left[-\beta \mathbf{E} \cdot\left(\mathbf{Q} \circ S_{\mathbf{E}}^{-t}-\mathbf{Q}\right)\right] .
$$

For any subset $\Delta$ of $M$ and time interval $I$ we may define the "product set" in the special flow coordinates $\Delta \times I=\bigcup_{\tau \in I} S_{\mathbf{E}}^{\tau}[\Delta]$ contained in $\mathfrak{M}$. Since the particle moves at the same speed $v$ under $S_{\mathbf{E}}^{t}$ for each $\mathbf{E}$, it follows that for a net $\left\{\Delta_{x}\right\}$ of Vitali sets converging to $x \in M$,

$$
\begin{aligned}
\frac{d\left(\dot{T}_{\mathbf{E}}^{-1} v_{0}\right)}{d v_{0}(x)} & =\lim _{\Delta_{x} \downarrow\{x\}} \frac{v_{0}\left(T_{\mathbf{E}}\left[\Delta_{x}\right]\right)}{v_{0}\left(\Delta_{x}\right)} \\
& =\lim _{\Delta_{x} \downarrow\{x\} \delta T \downarrow 0} \lim _{\delta T 0} \frac{\mu_{0}\left(S_{\mathbf{E}}^{\tau_{\mathbf{E}}(x)}\left(\Delta_{x} \times[-\delta T, \delta T]\right)\right)}{\mu_{0}\left(\Delta_{x} \times[-\delta T, \delta T]\right)} \\
& =\frac{d\left(\dot{S}_{\mathbf{E}}^{-\tau_{\mathbf{E}}(x)} \mu_{0}\right)}{d \mu_{0}(x)} \quad v_{0} \text {-a.e. } x .
\end{aligned}
$$

Thus we obtain

$$
\begin{aligned}
\frac{d\left(\dot{T}_{\mathbf{E}}^{-1} v_{0}\right)}{d v_{0}} & =\exp \left[-\beta \mathbf{E} \cdot\left(\mathbf{Q} \circ T_{\mathbf{E}}-\mathbf{Q}\right)\right] \\
& =\exp \left[-\beta \mathbf{E} \cdot \boldsymbol{\Delta}_{\mathbf{E}}\right] .
\end{aligned}
$$

The above argument is missing some details, but can be completed without great difficulty to give the final formula (20) for the Radon-Nikodým derivative. Observe from this equality that $v_{0}\left(\exp \left[-\beta \mathbf{E} \cdot \Delta_{\mathbf{E}}\right]\right)=1$. Therefore, finally

$$
\left.\hat{T}_{\mathbf{E}}^{n} v_{0}(\phi)=v_{0}(\phi)+\sum_{k=1}^{n} v_{0}\left[\left(\phi \circ T_{\mathbf{E}}^{k}\right)\left(1-e^{-\beta \mathbf{E} \cdot \Delta_{\mathbf{E}}}\right)\right)\right] .
$$

Now, if we assume that the measure $\hat{T}_{\mathbf{E}}^{n} v_{0}$ converges weakly as $n \rightarrow+\infty$ to $v_{\mathbf{E}}^{+}$and that the summation converges also in that limit for at least $C^{1}$-smooth $\phi$, then we obtain exactly

$$
v_{\mathbf{E}}^{+}(\phi)=v_{0}(\phi)+\sum_{n=1}^{\infty} v_{0}\left[\left(\phi \circ T_{\mathbf{E}}^{n}\right)\left(1-e^{-\beta \mathbf{E} \cdot \Delta_{\mathbf{E}}}\right)\right],
$$

for such $\phi$. If the convergence of the sum can be proven for a larger class of functions by some uniform summable bound on its terms, e.g. as below for $H_{\alpha}^{*}$, then the formula can be extended to that class also by approximation.

We now provide the calculation for the second half of Proposition 3, the nonlinear response formula. Since $\mathbf{v}=\dot{\mathbf{Q}}$, we see that

$$
\begin{aligned}
\mathbf{J}(\mathbf{E}) \equiv \mu_{\mathbf{E}}^{+}(\mathbf{v}) & =\frac{1}{\bar{\tau}_{\mathbf{E}}} \int_{M} \nu_{\mathbf{E}}^{+}(d x) \int_{0}^{\tau_{\mathbf{E}}(x)} d \tau \mathbf{v}(x, \tau) \\
& =\frac{1}{\bar{\tau}_{\mathbf{E}}} \nu_{\mathbf{E}}^{+}\left(\mathbf{Q} \circ T_{\mathbf{E}}-\mathbf{Q}\right) .
\end{aligned}
$$


Thus, if Eq. (21) is shown to hold for $\phi=\Delta_{\mathbf{E}} / \bar{\tau}_{\mathbf{E}}$, we may simply substitute to obtain the formula for $\mathbf{J}(\mathbf{E})$. However, it is somewhat more convenient to use the invariance to write

$$
\mathbf{J}(\mathbf{E})=\frac{1}{2 \bar{\tau}_{\mathbf{E}}} \nu_{\mathbf{E}}^{+}\left(\boldsymbol{\Delta}_{\mathbf{E}}+\tilde{\boldsymbol{\Delta}}_{\mathbf{E}}\right)
$$

with $\tilde{\Delta}_{\mathbf{E}} \equiv \Delta_{\mathbf{E}} \circ T_{\mathbf{E}}^{-1}=\mathbf{Q}-\mathbf{Q} \circ T_{\mathbf{E}}^{-1}$. In that case, the contribution from the first term of Eq. (21) is seen to vanish. Indeed, $v_{0}$ is invariant under time-inversion and also $\mathbf{Q}(\tilde{x})=\mathbf{Q}(x)$, so that $v_{0}\left(\mathbf{Q} \circ T_{\mathbf{E}}\right)=v_{0}\left(\mathbf{Q} \circ T_{\mathbf{E}}^{-1}\right)$. On the other hand, the two terms from the summation are easily calculated to give the response formula Eq. (17).

The next problem is to give arguments for the results on linear response in Proposition 4 . We define a remainder function

$$
R_{\mathbf{E}}(x) \equiv \frac{\left(1-\beta \mathbf{E} \cdot \Delta_{0}(x)-e^{-\beta \mathbf{E} \cdot \Delta_{\mathbf{E}}(x)}\right)}{|\mathbf{E}|}
$$

so that we may write

$$
v_{\mathbf{E}}^{+}(\phi)=v_{0}(\phi)+\beta \mathbf{E} \cdot \sum_{n=1}^{\infty} v_{0}\left[\left(\phi \circ T_{\mathbf{E}}^{n}\right) \boldsymbol{\Delta}_{0}\right]+|\mathbf{E}| \cdot \sum_{n=1}^{+\infty} v_{0}\left[\left(\phi \circ T_{\mathbf{E}}^{n}\right) R_{\mathbf{E}}\right] .
$$

Observe that $v_{0}\left(R_{\mathbf{E}}\right)=0$ for every $\mathbf{E}$. Furthermore, $R_{\mathbf{E}}$ is shown in Sect. (e) to be bounded uniformly in $\mathbf{E}$ and $\lim _{\mathbf{E} \rightarrow 0} R_{\mathbf{E}}(x)=0 v_{0}$-a.e. (by the $C^{2}$ convergence of $T_{\mathbf{E}}$ to $T_{0}$ for $\mathbf{E} \rightarrow 0$ ). From dominated convergence the terms in the last sum of Eq. (22) go individually to zero. Thus, only a summable bound on $v_{0}\left[\left(\phi \circ T_{0}^{n}\right) R_{\mathbf{E}}\right]$ uniform in $\mathbf{E}$ is required to infer that the last term is rigorously $o(|\mathbf{E}|)$. The argument for Ohm's law and the Einstein relation is made similarly. Note first that $\boldsymbol{\Delta}_{\mathbf{E}}$ is bounded (uniformly in $\mathbf{E}$ ) and $\lim _{\mathbf{E} \rightarrow 0} \boldsymbol{\Delta}_{\mathbf{E}}(x)=\boldsymbol{\Delta}_{0}(x) v_{0}$-a.s. Thus, $v_{0}\left[\boldsymbol{\Delta}_{\mathbf{E}}\left(1-e^{-\beta \mathbf{E} \cdot \boldsymbol{\Delta}_{\mathbf{E}}}\right)-\beta\left(\boldsymbol{\Delta}_{0} \otimes \boldsymbol{\Delta}_{0}\right) \cdot \mathbf{E}\right]=o(|\mathbf{E}|)$. Likewise, $v_{0}\left[\left(\boldsymbol{\Delta}_{\mathbf{E}}{ }^{\circ} T_{\mathbf{E}}^{n}\right)\left(1-e^{-\beta \mathbf{E} \cdot \boldsymbol{\Delta}_{\mathbf{E}}}\right)\right.$ $\left.-\left(\boldsymbol{\Delta}_{0} \circ T_{0}^{n}\right) \beta \mathbf{E} \cdot \boldsymbol{\Delta}_{0}\right]=o(|\mathbf{E}|)$ for each $n$ and a suitable uniform decay bound gives also $o(|\mathbf{E}|)$ for the summation. Thus, $\mathbf{J}=\boldsymbol{\sigma} \cdot \mathbf{E}+o(|\mathbf{E}|)$ with

$$
\boldsymbol{\sigma}=\beta\left(\frac{1}{2 \bar{\tau}_{0}} v_{0}\left(\boldsymbol{\Delta}_{0} \otimes \boldsymbol{\Delta}_{0}\right)+\frac{1}{\bar{\tau}_{0}} \sum_{n=1}^{+\infty} v_{0}\left(\left(\boldsymbol{\Delta}_{0} \circ T_{0}^{n}\right) \otimes \boldsymbol{\Delta}_{0}\right)\right),
$$

which is obviously equivalent to what is stated in Eq. (18) of Proposition 4.

We briefly indicate the modifications in the argument required if the magnetic field $\mathbf{B} \neq \mathbf{0}$. In that case, the derivations of the Radon-Nikodým derivative formula (20) and its consequence Eq. (21) are unchanged. However, the contribution to $\mathbf{J}(\mathbf{B}, \mathbf{E})$ from the first term in Eq. (21), namely, $\mathbf{J}^{0}(\mathbf{B}, \mathbf{E})=\frac{1}{2 \bar{\tau}_{\mathbf{B}, \mathbf{E}}}\left(v_{0}\left(\mathbf{Q} \circ T_{\mathbf{B}, \mathbf{E}}\right)-\right.$ $\left.v_{0}\left(\mathbf{Q} \circ T_{\mathbf{B}, \mathbf{E}}^{-1}\right)\right)$, is no longer zero, because $v_{0}\left(\mathbf{Q} \circ T_{\mathbf{B}, \mathbf{E}}\right)=v_{0}\left(\mathbf{Q} \circ T_{-\mathbf{B}, \mathbf{E}}^{-}\right)$is all that can now be inferred by the use of time-inversion. Nevertheless, $\mathbf{J}^{0}(\mathbf{B}, \mathbf{E})$ is easily seen to be $o(1)$ as $\mathbf{E} \rightarrow 0$ (since $S_{\mathbf{B}, 0}^{t}$ is Hamiltonian and preserves Liouville measure), while its contribution $\boldsymbol{\sigma}^{0}(\mathbf{B})$ to the conductivity $\boldsymbol{\sigma}(\mathbf{B})=\left.\frac{\partial}{\partial \mathbf{E}} \mathbf{J}(\mathbf{B}, \mathbf{E})\right|_{\mathbf{E}=0}$ may be checked to be finite, antisymmetric, and, by time-inversion, odd in $\mathbf{B}$. The other "dynamical" contribution, $\boldsymbol{\sigma}^{D}(\mathbf{B})$, obtained from the second term in Eq. (21) has the same form as the $\mathbf{B}=0$ expression above with $\bar{\tau}_{0} \rightarrow \bar{\tau}_{\mathbf{B}, 0}, \Delta_{0} \rightarrow \Delta_{\mathbf{B}, 0}$, and $T_{0} \rightarrow T_{\mathbf{B}, 0}$, and, 
furthermore, it obeys $\boldsymbol{\sigma}^{D}(\mathbf{B})^{\top}=\sigma^{D}(-\mathbf{B})$ by using time-reversal. The total conductivity $\boldsymbol{\sigma}(\mathbf{B})=\sigma^{0}(\mathbf{B})+\sigma^{D}(\mathbf{B})$ then also obeys the relation $\boldsymbol{\sigma}(\mathbf{B})^{\top}=\boldsymbol{\sigma}(-\mathbf{B})$ (Onsager reciprocity) and its symmetric part $\tilde{\boldsymbol{\sigma}}(\mathbf{B})$, whose entire contribution is "dynamical," is equal to

$$
\tilde{\boldsymbol{\sigma}}(\mathbf{B})=\beta\left(\frac{1}{2 \bar{\tau}_{\mathbf{B}, 0}} \sum_{n=-\infty}^{+\infty} v_{0}\left(\left(\boldsymbol{\Delta}_{\mathbf{B}, 0} \circ T_{\mathbf{B}, 0}^{n}\right) \otimes \boldsymbol{\Delta}_{\mathbf{B}, 0}\right)\right) .
$$

This last equation can be trivially rewritten as $\tilde{\boldsymbol{\sigma}}(\mathbf{B})=\beta \cdot \mathbf{D}(\mathbf{B})$, and the matrix $\mathbf{D}(\mathbf{B})$ which appears there has another physical interpretation. If one follows the arguments of references $[3,5$, and 9], one can show that in the case $\mathbf{B} \neq 0$ (but small enough), a rescaled particle trajectory $\mathbf{X}_{\mathbf{B}}^{\varepsilon}(t)$ converges weakly to the solution of the stochastic differential equation $d \mathbf{X}_{\mathbf{B}}(t)=\left(\mathbf{X}_{\mathbf{B}}(t) \times \mathbf{B}\right) d t+d \mathbf{W}_{\mathbf{B}}(t), \mathbf{X}_{\mathbf{B}}(0)=0$, where $\mathbf{W}_{\mathbf{B}}(t)$ is the two-dimensional Brownian process with covariance $\mathbf{D}(\mathbf{B})$. Hence, $\mathbf{D}(\mathbf{B})$ is the diffusion matrix in the presence of the magnetic field and the result $\tilde{\boldsymbol{\sigma}}(\mathbf{B})=\beta \cdot \mathbf{D}(\mathbf{B})$ is a natural generalization of the usual Einstein relation.

(b) Existence of Local Stable and Unstable Manifolds. We need here a few additional notations. Set $S_{n}=T_{\mathbf{E}}^{n-1} S_{1}$ and $S_{-n}=T_{\mathbf{E}}^{-(n-1)} S_{-1}$ for every $n \geqq 1$. A natural measure equivalent to the length on a smooth curve $\gamma \in M$ is defined as

$$
\rho(\gamma)=\int_{\gamma} \cos \varphi d r
$$

in terms of the standard coordinates $(r, \phi)$, see $[4,5,15]$.

Lemma 1 (Stable and unstable fibers). Almost every point $x \in M$ (with respect to the measure $v_{0}$ ) has stable and unstable fibers, denoted by $\gamma^{s}(x)$ and $\gamma^{u}(x)$, respectively, passing through $x$.

Proof. First we observe that the billiard system $\left\{S_{0}^{t}\right\}$ has here a finite horizon and a smooth strictly concave boundary $\partial Q$. In particular, the time of first return $\tau_{0}(x)$ is bounded away from 0 and $\infty: 0<\tau_{\min } \leqq \tau_{0}(x) \leqq \tau_{\max }<\infty$. These properties lead to a strong hyperbolicity of the billiard map $T_{0}$. The hyperbolicity of $T$ can be defined in terms of families of strictly invariant cones which are popular nowadays, see e.g. $[40,6,11]$. These are two families of cones in the tangent planes $\mathscr{T}_{X} M$ to the manifold $M$ such that the unstable cones are strictly invariant under $D T_{0}$ while the stable ones are strictly invariant under $D T_{0}^{-1}$. Note that the rate of expansion (contraction) of each tanget vector in the unstable (stable) cones in our $\rho$-metric is bounded away from 1 , see e.g. $[4,5]$. We denote the minimal (maximal) rate of expansion (contraction) by $W_{0}>1$ (resp., $w_{0}<1$ ). Due to the smallness of $\mathbf{E}$ the same cones are still invariant under $D T_{\mathbf{E}}$ (respectively, under $D T_{\mathbf{E}}^{-1}$ ) and the minimal (maximal) rate of expansion (contraction) $W_{\mathbf{E}}\left(w_{\mathbf{E}}\right)$ are close to $W_{0}\left(w_{0}\right)$ and still bounded away from 1 . Nevertheless, we cannot apply here the usual theorems on invariant cones [40] since no invariant measure has been constructed yet for $T_{\mathbf{E}}, \mathbf{E} \neq 0$. Instead, we apply a direct method for constructing stable and unstable fibers, see $[34,35,4]$.

For any point $x \in M$ and $n \geqq 1$ we take a curve $\gamma_{n}^{\prime}$ passing through $T^{-n} x$ and lying in unstable cones. (This means that at every point of that curve the tangent vector to the curve belongs to the unstable cone.) Then $\lim _{n \rightarrow \infty} T^{n} \gamma_{n}^{\prime}$ gives us the unstable fiber $\gamma_{x}^{u}$ provided that $T^{n}$ is continuous on $\gamma_{n}^{\prime}$ for all $n$ and the length of $T^{n} \gamma_{n}^{\prime}$ is bounded away from 0 . To estimate that length one needs the bound

$$
v_{0}\left(U_{\varepsilon}\left(S_{ \pm 1}\right)\right) \leqq c_{1} \varepsilon
$$


for all $\varepsilon>0$, where $U_{\varepsilon}\left(S_{ \pm 1}\right)$ denotes the $\varepsilon$-neighborhood of $S_{ \pm 1}$. The estimate (23) readily comes from the fact that in case of finite horizon the set $S_{ \pm 1}$ is always a finite union of smooth compact curves in $M$. Standard arguments $[34,35,4]$ show that $\gamma^{u}(x)$ has the $\rho$-length $\geqq \varepsilon_{0}$ as soon as $T^{-n} x$ lies outside $U_{\varepsilon_{n}}\left(S_{ \pm 1}\right)$, $\varepsilon_{n}=\varepsilon_{0} \cdot w_{\mathrm{E}}^{n}$ for all $n \geqq 1$. Therefore, the set of points $x \in M$ with the unstable fiber $\gamma^{u}(x)$ of length $<\varepsilon_{0}$ has the $v_{0}$-measure less then

$$
\sum_{n=0}^{\infty} v_{0}\left(T_{\mathbf{E}}^{n} U_{\varepsilon_{n}}\left(S_{ \pm 1}\right)\right)
$$

The map $T_{\mathbf{E}}$ can contract or expand the measure $v_{0}$ but with a rate not greater than $\exp (\omega|\mathbf{E}|)$, where $\omega=p \beta \tau_{\max } / m$ is a constant, see Sect. (a) and [30]. For small $|\mathbf{E}|$ this rate is close to 1 and therefore less than $w_{\mathbf{E}}^{-1}$. The sum (24) then does not exceed

$$
c_{2} \varepsilon_{0} \sum_{n=1}^{\infty}(\exp (\omega|\mathbf{E}|))^{-n} w_{\mathbf{E}}^{n}=c_{3} \varepsilon_{0}
$$

Hence the lemma.

Corollary 2. The $v_{0}$-measure of the set of points $x \in M$ for which the $\rho$-length of the unstable (stable) fiber is less than $\varepsilon$ does not exceed $c_{4} \cdot \varepsilon$.

Remark. In the proof of Lemma 1 we have explicitly found the necessary bound on $|\mathbf{E}|: \exp \left[|\mathbf{E}| p \beta \tau_{\max } / m\right]<w_{\mathbf{E}}^{-1}$. However, in our further considerations we can no longer do so.

Corollary 3. For almost every point $X$ in the whole space $\mathfrak{M}$ (with respect to the measure $\mu_{0}$ ) there are stable and unstable manifolds of the flow $\left\{S_{\mathbf{E}}^{t}\right\}$ passing through $X$.

Proof. Due to Lemma 1 there is a stable curve $\gamma^{s}(x)$ for $v_{0}$-almost every $x \in M$. It certainly provides a bunch of trajectories which converges exponentially fast in the future. Next, for any point $y \in \gamma^{s}(x)$ denote $\theta_{n}(y)=\tau(y)+\tau\left(T_{\mathbf{E}} y\right)+\cdots$ $+\tau\left(T_{\mathbf{E}}^{n-1} y\right)$ the time up to the $n^{\text {th }}$ reflection. It is now clear that $\left|\theta_{n}(y)-\theta_{n}(x)\right| \leqq \sum_{0}^{n-1}\left|\tau\left(T_{\mathbf{E}}^{i} y\right)-\tau\left(T_{\mathbf{E}}^{i} x\right)\right| \leqq c_{5} \cdot \operatorname{dist}(x, y)$. Therefore, the function $\Delta(y)=\lim _{n \rightarrow \infty}\left(\theta_{n}(y)-\theta_{n}(x)\right)$ is continuous on $\gamma^{s}(x)$ and has a finite derivative at $x$ with respect to the $\rho$-length. Now the map $y \mapsto S^{\Delta(y)} y$ transforms the fiber $\gamma^{s}(x)$ into the stable manifold for the flow $\left\{S_{\mathbf{E}}^{t}\right\}$. The projection of that manifold into $Q$ is a curve transversal to the trajectories of the flow $\left\{S_{\mathbf{E}}^{t}\right\}$. Note that generally that curve is not orthogonal to the trajectories of the flow, as it was in the case of billiards.

In the case of billiard flow $\left\{S_{0}^{t}\right\}$ the curvature of a stable (unstable) manifold at a point $X \in \mathfrak{M}$ is expressed through a continued fraction $B^{s}(X)$ (resp., $B^{u}(X)$ ), see $[34,36]$. The differential equation of the stable (unstable) fibers in $M$ is then readily obtained as

$$
\frac{d \varphi}{d r}=-B^{s}(x) \cos \varphi+\kappa(x) \quad\left(\frac{d \varphi}{d r}=B^{u}(x) \cos \varphi+\kappa(x)\right),
$$

where $\kappa(x)$ stands for the (positive) curvature of the boundary $\partial Q$ at the point $x$. In our model, with $|\mathbf{E}| \neq 0$, the curvature of stable or unstable manifold is no longer expressed through any continued fraction. But, if we denote by $B_{\mathbf{E}}^{s}(X)\left(B_{\mathbf{E}}^{u}(X)\right)$ the 
curvature of the orthogonal section of the beam of trajectories generated by the stable (unstable) manifold at $X \in \mathfrak{M}$, then the expressions (25) remain true for $|\mathbf{E}| \neq 0$.

Lemma 4 (Absolute continuity). The stable and unstable fibers in the space $M$ are absolutely continuous with respect to the measure $v_{0}$.

The statement of Lemma 4 means that the canonical isomorphism on stable and unstable fibers, see e.g. $[2,5]$, is absolutely continuous with respect to the $\rho$-length on those fibers. The proof of Lemma 4 goes the same way as that of its analogue for billiards $[34,15]$ and we do not go into detail.

Remark 5 (Alignment). The images $S_{n}$ of the singularity curves lie in unstable cones for $n>0$ and in stable cones for $n<0$. Thus, they become almost parallel to unstable fibers as $n \rightarrow \infty$ and to stable fibers as $n \rightarrow-\infty$.

We do not make this statement more precise.

(c) Homogeneous Fibres and Markov Sieves. Stable and unstable curves with the absolute continuity property constitute the main tool for the study of ergodic properties of hyperbolic systems. But the exploration of their statistical properties requires the so-called homogeneous fibers. These fibers have been first introduced for billiards in [5]. As it was explained there, the billiard map $T_{0}$ expands unstable manifolds but nonuniformly: the rate of expansion grows in the neighborhood of $\partial M$ where $\cos \varphi$ vanishes. In order to control this rate the authors of [5] split the neighborhood of $\partial M$ into a countable number of strips the thinner the closer to $\partial M$. The strips were defined by the equations $\pi / 2-(n+1)^{-\eta} \geqq \varphi \geqq \pi / 2-n^{-\eta}$ in the neighborhood of the line $\varphi=\pi / 2$ and $-\pi / 2+(n+1)^{-\eta} \leqq \varphi \leqq-\pi / 2+n^{-\eta}$ in the neighborhood of the line $\varphi=-\pi / 2$, where $n \geqq n_{0}$. The parameters $\eta>1$ and $n_{0} \geqq 1$ are rather arbitrary except $n_{0}$ should be large enough. We denote $\mathfrak{D}_{0}$ the union of the lines separating the strips.

Definition. An unstable (stable) fiber $\gamma^{u}\left(\gamma^{s}\right)$ is said to be homogeneous (or 0-homogeneous) if its images $T^{-n} \gamma^{u}\left(T^{n} \gamma^{s}\right)$ for $n \geqq 0$ never cross $\mathfrak{D}_{0}$, the borders of the above strips. An unstable (stable) fiber $\gamma^{u}\left(\gamma^{s}\right)$ is said to be $m$-homogeneous, $m \geqq 1$, if its larger preimage $T^{m} \gamma^{u}\left(T^{-m} \gamma^{s}\right)$ is a homogeneous fiber.

The following lemmas have been proved in full detail for billiards in [5]. For small $|\mathbf{E}|$ they are also valid for our system and the proofs are essentially the same.

Lemma 6 (Existence). Almost every point $x \in M$ (with respect to $v_{0}$ ) has homogeneous stable and unstable fibers passing through $x$.

The largest smooth components of the homogeneous stable and unstable fibers passing through $x$ are denoted $\gamma^{0 s}(x)$ and $\gamma^{0 u}(x)$ respectively. The next lemma is a natural extension to Corollary 2 .

Lemma 7 (Distribution of length). For every $\varepsilon>0$ the set of points $x \in M$ with the homogeneous fibers of length $<\varepsilon$ has $v_{0}$-measure less than $c_{6} \varepsilon^{a_{1}}$, where $a_{1}$ depends on the choice of the value of $\eta$ above.

For every point $x \in M$ and $k \geqq 1$ denote by $w_{k}^{u}(x)$ the rate of contraction of $\gamma^{u}(x)$ at the point $x$ under $T^{-k}$. 
Lemma 8 (Homogeneity). Let $\gamma^{0 u}$ be an arbitrary m-homogeneous unstable fiber, $m \geqq 0$. Then for every pair $x, y \in \gamma^{0 u}$ and every $k \geqq 1$

$$
\left|\frac{w_{k}^{u}(x)}{w_{k}^{u}(y)}-1\right| \leqq c_{7} \alpha_{1}^{m},
$$

where $c_{7}, \alpha_{1}$ are determined by $\eta$ and $n_{0}$ above.

Our further considerations extensively use elements of Markov partitions for hyperbolic systems. In our notions and notations we follow the traditions of works $[2,4,5]$. A basic notion in the theory of Markov partitions is a parallelogram. It is defined as a subset $A \subset M$ such that for any two points $x, y \in A$ the point $z=\gamma^{u}(x) \cap \gamma^{s}(y)$ exists and again belongs to $A$. If we substitute $\gamma^{0 u}(x)$ and $\gamma^{0 s}(y)$ for $\gamma^{u}(x)$ and $\gamma^{s}(y)$ in this definition, we obtain the definition of a homogeneous parallelogram. If for a parallelogram $A$ both its images $T^{m} A$ and $T^{-m} A$ are homogeneous parallelograms, then $A$ is said to be $m$-homogeneous. In what follows we always consider only homogeneous parallelograms without specifying this.

Any parallelogram $A$ is a Cantor set with a grid structure. We denote $\gamma_{A}^{u, s}(x)=\gamma^{u, s}(x) \cap A$ for every $x \in A$. The sets $\gamma_{A}^{u}(x)$ (and $\gamma_{A}^{s}(x)$ ) for all $x \in A$ are Cantor sets on the corresponding fibers which are canonically isomorphic, see e.g. [5].

Let $A_{0}$ be an $m$-homogeneous parallelogram and $x_{0} \in A_{0}$. As shown in [5], the $v_{0}$-measure of any subparallelogram $A \subset A_{0}$ can be approximated by the value

$$
v_{0}^{a}(A)=\rho\left(\Gamma_{A}^{u}\right) \rho\left(\Gamma_{A}^{s}\right)\left(B^{u}\left(x_{0}\right)+B^{s}\left(x_{0}\right)\right) .
$$

Here $\Gamma_{A}^{u, s}$ denote the images of $A$ on the fibers $\gamma^{u, s}\left(x_{0}\right)$ under the canonical isomorphisms. More precisely, the value (26) is an approximation to the $v_{0}$ measure of $A$, constructed below, with an exponentially small error:

$$
\left|v_{0}^{a}(A) / v_{0}(A)-1\right| \leqq c_{8} \alpha_{2}^{m} .
$$

Evidently, the image $T_{\mathrm{E}}^{n} A$ of a parallelogram $A$ is a finite union of parallelograms. Consequently, the intersection $T_{\mathbf{E}}^{n} A \cap B$ is again a finite union of parallelograms, where $B$ is another parallelogram. We say that a subparallelogram $C \subset B$ is $u$-inscribed (s-inscribed) in $B$ if $\gamma_{C}^{u}(x)=\gamma_{B}^{u}(x)$ (resp. $\gamma_{C}^{s}(x)=\gamma_{B}^{s}(x)$ ) for every $x \in C$. Of the parallelograms composing $T_{\mathbf{E}}^{n} A \cap B$, the union of those $u$-inscribed in $B$ and such that their images under $T_{\mathbf{E}}^{-n}$ are $s$-inscribed in $A$ is called the regular part and denoted by $\mathfrak{R}\left(T_{\mathbf{E}}^{n} A \cap B\right)$, while the union of the others is called the irregular part of that intersection and denoted by $\mathfrak{I}\left(T_{\mathbf{E}}^{n} A \cap B\right)$. Dual notations are introduced for $T_{\mathbf{E}}^{n} A \cap B$ with $n \leqq-1$. The intersection $T_{\mathbf{E}}^{n} A \cap B$ is said to be regular if it contains no irregular part.

The Markov partition for $T_{\mathbf{E}}$ is a countable partition (mod 0$)$ of the manifold $M$ into parallelograms $\left\{A_{1}, A_{2}, \ldots\right\}$ such that the intersections $T^{n} A_{i} \cap A_{j}$ are regular for any pair $A_{i}, A_{j}$ and any $n \neq 0$. Note that there cannot be finite Markov partitions due to the presence of arbitrary short fibers. Markov partitions for $T_{0}$ have been constructed in $[2,4]$. This construction can be extended to $T_{\mathbf{E}}$ with small $|\mathbf{E}| \neq 0$. However, it seems to be of no use for us because it is not clear whether the parallelograms of the Markov partition cover a.e. point in $M$ with respect to the needed measure $v_{\mathbf{E}}^{+}$.

We use Markov sieves introduced in [5, 8] and defined below. They consist of a finite number of parallelograms and therefore do not cover a set of full measure in 
$M$. But the Markov sieve turns out to be much easier to construct and to control than the Markov partition, and it also yields useful estimates of the statistical properties of hyperbolic dynamical systems with singularities $[5,8]$. Let us stress also that the Markov sieves depend on the interval of time which is considered.

The Markov sieves are closely related to the pre-Markov partitions $[4,5,8]$ and we define them both below.

Any domain $\Pi$ in $M$ bounded by two unstable and two stable fibers is called the quadrilateral. Its boundary $\partial \Pi$ consists of two unstable fibers called the $u$-sides of $\Pi$ and two stable ones called the s-sides of $\Pi$. The union of two $u$-sides is denoted by $\partial^{u} \Pi$ and that of two $s$-sides is denoted by $\partial^{s} \Pi$. Fix a sufficiently large $m \geqq 1$ and let $\varepsilon>0$ be arbitrarily small and real $\left(\varepsilon<\varepsilon_{0}(m)\right)$. A pre-Markov partition for the map $T^{m}$ is a finite partition $\xi_{0}=\xi_{0}(\varepsilon)$ of $M$ into curvilinear polygons $P_{1}, \ldots, P_{k}$ whose properties are listed next: The boundary $\partial \xi_{0}=\bigcup \partial P_{i}$ is the union of $S_{-m, m}=\bigcup_{k=-m}^{m} S_{k}$ and a finite collection of unstable and stable fibers. Respectively, we denote $\partial \xi_{0}=\partial^{0} \xi_{0} \cup \partial^{u} \xi_{0} \cup \partial^{s} \xi_{0}$, where $\partial^{0} \xi_{0}=S_{-m, m}$ and $\partial^{u} \xi_{0}\left(\partial^{s} \xi_{0}\right)$ consists of unstable (stable) fibers. The main properties of $\xi_{0}$ are $T\left(\partial^{s} \xi_{0}\right) \subseteq \partial^{s} \xi_{0}$ and $T^{-1}\left(\partial^{u} \xi_{0}\right) \subseteq \partial^{u} \xi_{0}$. All the interior angles of the polygons $P \in \xi_{0}$ both sides of which are unstable and stable fibers are always less than $\pi$. The sides of the polygons $P \in \xi_{0}$ lying on unstable (stable) fibers are less than $c_{9} \varepsilon$ and their images under $T^{m}$ (resp., $T^{-m}$ ) remain less than $c_{10} \varepsilon$. If a polygon $P_{i} \in \xi_{0}$ does not touch the set $S_{-m, m}$, then it is a quadrilateral. All the other elements of $\xi_{0}$ form a neighborhood of $S_{-m, m}$ which we call the necklace and denote by $\mathfrak{N}\left(\xi_{0}\right)$. The necklace is actually contained in a $c_{11} \sqrt{\varepsilon}$-neighborhood of $S_{-m, m}$, and so its $v_{0}$-measure is less than $c_{12} \varepsilon^{a_{2}}$. We also define an extended necklace $\mathfrak{N}_{e}\left(\xi_{0}\right)$ as the union of $\mathfrak{N}\left(\xi_{0}\right)$ and all the quadrilaterals $\Pi \in \xi_{0}$ intersecting $\mathfrak{D}_{0}$, the borders of the strips constructed in the definition of homogeneous fibers. It is easily checked that $v_{0}\left(\mathfrak{N}_{e}\left(\xi_{0}\right)\right) \leqq c_{13} \varepsilon^{a_{3}}$.

Remark 9. Every stable and unstable fiber is either transversal to $S_{-m, m}$ or tangent to it, and in the latter case the tangency has the order two, see $[34,2]$. Therefore, the necklace $\mathfrak{N}\left(\xi_{0}\right)$ can cover only a small part of that fiber so that the total $\rho$-length of that part is less than $c_{14} \varepsilon^{a_{4}}$. The extended necklace $\mathfrak{N}_{e}\left(\xi_{0}\right)$ also has that property. Likewise, the $\varepsilon$-neighborhood of $S_{-m, m}$ for any $\varepsilon>0$ covers only a small part of that fiber so that the total length of that part is less than $c_{15} \varepsilon^{a_{5}}$.

For precise description of the evolution of parallelograms in $M$ we use the following geometrical notions introduced in $[5,2]$. For any parallelogram $A$ the minimal closed quadrilateral containing $A$ is called the support of $A$ and denoted by $\Pi(A)$. We say that a segment of an unstable (stable) fiber is inscribed in a quadrilateral $\Pi$ if it lies within $\Pi$ and terminates on two $s$-sides ( $u$-sides) of $\Pi$. A parallelogram $A$ is said to be maximal if it intersects all the unstable and stable fibers inscribed in its support $\Pi(A)$. In other words, to construct a maximal parallelogram one should take a quadrilateral $\Pi$, draw all the unstable and stable fibers inscribed in $\Pi$ and take all the mutual intersections; thus the maximal parallelogram would consist of the points of intersections of these fibers. The parallelogram so obtained is denoted by $A(\Pi)$.

Now let $n \geqq 1$ be a large number and $\varepsilon_{n}=\alpha_{3}^{n}$ for some $\alpha_{3} \in(0,1)$. Consider the partition $\xi_{n}=T^{-n} \xi_{0} \vee T^{-n+1} \xi_{0} \vee \ldots \vee T^{n} \xi_{0}$ of the space $M$, where $\xi_{0}=\xi_{0}\left(\varepsilon_{n}\right)$ is a pre-Markov partition. Denote $\mathfrak{N}_{e}\left(\xi_{n}\right)=T^{-n} \mathfrak{N}_{e}\left(\xi_{0}\right) \cup \ldots \cup T^{n} \mathfrak{N}_{e}\left(\xi_{0}\right)$. Clearly, $v_{0}\left(\mathfrak{N}_{e}\left(\xi_{n}\right)\right) \leqq c_{16} \alpha_{4}^{n}$ for some $\alpha_{4} \in(0,1)$. Every element $\Pi$ of $\xi_{n}$ which lies outside $\mathfrak{R}_{e}\left(\xi_{n}\right)$ is a quadrilateral. Moreover, its images $T^{i} \Pi$ for $|i| \leqq n$ do not 
intersect $S_{-m, m}$ or $\mathfrak{D}_{0}$. Let $\Pi_{1}, \ldots, \Pi_{I}$ be all the elements of $\xi_{n}$ lying outside $\mathfrak{N}\left(\xi_{n}\right)$. The maximal parallelograms $A_{1}=A\left(\Pi_{1}\right), \ldots, A_{I}=A\left(\Pi_{I}\right)$ form the Markov sieve which we denote $\mathfrak{6}_{n}$. The properties of the partition $\xi_{n}$ and Lemma 7 ensure that $v_{0}\left(M \backslash \bigcup A_{i}\right) \leqq c_{17} \alpha_{5}^{n}$ for some $\alpha_{5} \in(0,1)$. All the parallelograms $A \in \mathbb{6}_{n}$ are maximal and $n$-homogeneous. Note that if an unstable fiber $\gamma_{1}^{u}$ crosses both $s$-sides of an element $A \in \mathfrak{G}_{n}$, then it intersects $A$ itself. If a fiber $\gamma_{1}^{u}$ intersects no parallelograms $A \in \mathfrak{G}_{n}$, then it is either too short (i.e. $\rho\left(\gamma_{1}^{u}\right) \leqq c_{18} \alpha_{6}^{n}$ for some $\left.\alpha_{6} \in(0,1)\right)$ or it lies mostly in $\mathfrak{N}_{e}\left(\xi_{n}\right)$. In the latter case one of its images $T^{i} \gamma_{1}^{u}$ for some $|i| \leqq n$ belongs to the extended necklace $\mathfrak{N}_{e}\left(\xi_{0}\right)$.

(d) Existence of the Invariant Measure. We now turn to the construction of the limit measure $v_{\mathbf{E}}^{+}$which is defined as the limit of $\hat{T}_{\mathbf{E}}^{n} v_{0}$ as $n \rightarrow \infty$. The conditional measure induced by $v_{0}$ on a segment of an unstable fiber $\gamma^{u}$ can be constructed as follows. For $n \geqq 1$ take a uniform probabilistic measure (with respect to the $\rho$-length) on the preimage $T^{-n} \gamma^{u}$ and then pull it back onto $\gamma^{u}$. The limit of the resulting measure on $\gamma^{u}$ as $n \rightarrow \infty$ gives the conditional measure on $\gamma^{u}$. Lemma 8 assures that the density of the conditional measure on any homogeneous unstable fiber is uniformly bounded away from 0 and $\infty$.

Let $\gamma^{u}$ be a homogeneous unstable fiber and $p^{c}$ denote the conditional absolutely continuous probability measure on $\gamma^{u}$ constructed above. It is now clear that the existence of the measure $v_{\mathbf{E}}^{+}$is equivalent to the fact that the limit of $p_{n}^{c}=\hat{T}_{\mathbf{E}}^{n} p^{c}$ as $n \rightarrow \infty$ exists and is independent of $\gamma^{u}$. This limit thus produces the measure $v_{\mathbf{E}}^{+}$itself. Note that the methods of [42] can give a weaker result, i.e. the existence of $\lim _{n \rightarrow \infty} n^{-1}\left(v_{0}+\hat{T}_{\mathbf{E}} v_{0}+\cdots+\hat{T}_{\mathbf{E}}^{n-1} v_{0}\right)$.

The measure $p_{n}^{c}$ for finite $n$ is concentrated on the image $T^{n} \gamma^{u}$ which is a finite or countable union of homogeneous unstable fibers. These fibers are called homogeneous components of $T^{n} \gamma^{u}$, see [5], or just components, for brevity. The structure and the distribution of those components in the space $M$ play the key role in our further considerations. The necessary properties of the components are accumulated in the next several lemmas. These lemmas have been first established for billiards in [5] and then for piecewise linear toral maps in [8].

For any $D>0$ and $n \geqq 1$ denote $\Gamma_{n, D}^{u}$ the union of all components of $T^{n} \gamma^{u}$ which have $\rho$-length $\geqq D$.

Lemma 10 (From short to long components). There is $D>0$ not depending on $n$ such that for any $n \geqq 1$,

$$
p^{c}\left(\gamma^{u} \backslash \bigcup_{k=1}^{n} T^{-k} \Gamma_{k, D}^{u}\right) \leqq c_{19} \alpha_{7}^{n} / \rho\left(\gamma^{u}\right)
$$

with some $c_{19}, \alpha_{7}$ determined by $\eta$ and $n_{0}$.

The meaning of the lemma is that during the first $n$ iterates of $T$, if $n \geqq$ $-c_{20} \ln \rho\left(\gamma^{u}\right)$, the majority of points $x \in \gamma^{u}$ appear at least once in long components (of length $\geqq D$ ) of the images $T^{k} \gamma^{u}, 1 \leqq k \leqq n$.

The proof of Lemma 10 is based solely on the hyperbolic properties of the underlying map. It has been carried out in detail in $[5,8]$ and applies to our system, too.

Lemma 11 (Distribution of lengths of components). For any $\varepsilon>0$ and $n \geqq-c_{21} \ln \rho\left(\gamma^{u}\right)$ we have $p_{n}^{c}\left(\Gamma_{n, \varepsilon}^{u}\right) \geqq 1-c_{22} \varepsilon$.

Proof. Lemma 11 is just a stronger version of Lemma 10, but it is new and so we outline its proof here. The billiard map possesses the following basic property: for 
every $m \geqq 1$ the number of smooth components of $S_{-m, m}$ meeting at a single point of $M$ cannot exceed $K_{0} m$, where $K_{0}$ is a constant, see [4], Sect. 8. As a result for every $m \geqq 1$ there is $\varepsilon_{0}(m)>0$ such that any unstable fiber of length $\leqq \varepsilon_{0}(m)$ can cross at most $K_{0} m$ curves of $S_{-m, 0}$. This property is certainly valid for the map $T_{\mathbf{E}}$ for small $\mathbf{E},|\mathbf{E}|<E_{0}(m)$. Now we fix $m$ sufficiently large, so that $W_{\mathbf{E}}^{m} \gg K_{0} m$. Thus the image $T_{\mathrm{E}}^{m} \gamma_{1}^{u}$ of any short fiber $\gamma_{1}^{u}$ of length $\leqq \varepsilon_{0}(m)$ consists of at most $K_{0} m+1$ components and their total length is at least $\bar{\Lambda}_{\mathbf{E}}^{m}$ times greater than that of $\gamma_{1}^{u}$. Similar estimates can be carried out for homogeneous components, i.e. if we take into account the splitting of the components by the borderlines of the strips defined above. The technique used for obtaining those estimates is the same as in proof of Lemma 7, see [5] for details. Now we introduce a function $r_{n}(x)$ on $T_{\mathbf{E}}^{n} \gamma^{u}$ by $r_{n}(x)=\left\{\rho\right.$-distance from $x$ to the nearest endpoint of the component of $T_{\mathbf{E}}^{n} \gamma^{u}$ containing $x\}$. Note that $r_{n}(x)$ is actually smaller than the length of the component of $T_{\mathbf{E}}^{n} \gamma^{u}$, containing $x$. The above reasonings show that the distribution of $r_{n}(x)$ cannot concentrate near 0 , i.e. $p_{n}^{c}\left\{r_{n}(x)<\varepsilon\right\} \leqq c_{23} \varepsilon$. Hence the lemma.

Since we have not yet proved the existence of $\nu_{\mathbf{E}}^{+}$, we denote by $v_{\mathbf{E}}^{U}(B)$ and $\nu_{\mathbf{E}}^{L}(B)$ the upper and lower limits, respectively, of the sequence $\left\{p_{n}^{c}(B)\right\}_{0}^{\infty}$ for any measurable set $B$. The values $v_{\mathbf{E}}^{\mathrm{U}}, \mathrm{L}(B)$ may also depend on the choice of the initial fiber $\gamma^{u}$.

As an immediate consequence of Lemma 11 we obtain that the limit measure $v_{\mathbf{E}}^{+}$(if it exists) is nonatomic. In terms of $v_{\mathbf{E}}^{U, L}$ this means that $\lim _{\delta \rightarrow 0} v_{\mathbf{E}}^{U}\left(V_{\delta}(x)\right)=0$ for any point $x \in M, V_{\delta}(x)$ being here the $\delta$-disc centered at $x$. The following remark is a stronger version of this property:

Remark 12 (Nonatomic structure). For any unstable fiber $\tilde{\gamma}^{u}$ we have $\lim _{\delta \rightarrow 0} v_{\mathbf{E}}^{U}\left(V_{\delta}\left(\tilde{\gamma}^{u}\right)\right)=0$, where $V_{\delta}(\cdot)$ now denotes the $\delta$-neighborhood.

Next, let $\gamma^{u}$ be an unstable fiber of length $D / 2$ and $\Pi$ be a quadrilateral in $M$. Denote $\Gamma_{n, \Pi}^{u}$ the union of all subfibers in $T_{\mathbf{E}}^{n}\left(\gamma^{u}\right)$ which are $u$-inscribed in $\Pi$.

Lemma 13 (From long components into a fixed quadrilateral). There exists a quadrilateral $\Pi$ such that $v_{0}(A(\Pi))>0$ and constants $n_{1}>0, \beta_{1} \in(0,1)$ such that $p_{n}^{c}\left(\Gamma_{n, \Pi}^{u}\right) \geqq \beta_{1}$ for every $n \geqq n_{1}$. Here $n_{1}$ and $\beta_{1}$ are independent of $\gamma^{u}$ and of the field E.

Proof. The proof of Lemma 13 for billiards is based on the mixing property. Here we do not have it, so the arguments should be modified. For billiard map $T_{0}$ the statement of Lemma 13 has been proven [5] for any quadrilateral $\Pi$ such that $v_{0}(A(\Pi))>0$ and any unstable fiber $\gamma^{u}$ of length $\geqq D$. The proof is easily modified if, instead of the fiber $\gamma^{u}$, we take any curve $\tilde{\gamma}^{u}$ of length $\geqq D$ which is sufficiently close to unstable fibers (to be specific, such that $T_{0}^{-m}$ is smooth on $\tilde{\gamma}^{u}$ and $T_{0}^{-m} \tilde{\gamma}^{u}$ lies in unstable cones, $m$ being a large constant). Now we take an unstable fiber $\gamma^{u}$ of length $\geqq D$ for $T_{\mathbf{E}},|\mathbf{E}| \neq 0$. For small $|\mathbf{E}|$, a bit smaller part of $\gamma^{u}$ (of length $\geqq D-\varepsilon$ ) is certainly a curve close to unstable fibers in the above sense. The image $T_{\mathbf{E}}^{n} \gamma^{u}$ is close to $T_{0}^{n} \gamma^{u}$ for all $n \leqq n_{2}$ due to the smallness of $|\mathbf{E}|$, and $n_{2}$ here is large for small $|\mathbf{E}|$. Therefore the statement of the lemma follows for all $n, n_{1} \leqq n \leqq n_{2}$, with maybe smaller values of $D$ and $\beta_{1}$ than in the case of the billiard map $T_{0}$. To prove Lemma 13 for larger values of $n$, i.e. for $n \geqq n_{2}$, we observe that due to Lemmas 10,11 there are enough components of length $\geqq D$ in the images $T_{\mathbf{E}}^{n} \gamma^{u}$ for $1 \leqq n \leqq n_{2}-n_{1}$. To each of those components we apply the above reasonings again, etc. Thus we extend our estimate for all $n \geqq n_{1}$. 
Corollary 14. $v_{\mathbf{E}}^{L}(\Pi)>$ const $>0$ for any initial fiber $\gamma^{u}$. Note also that each fiber $\gamma_{1}^{u} u$-inscribed in $\Pi$ intersects the parallelogram $A=A(\Pi)$ and $\rho\left(\gamma_{1}^{u} \cap A\right)$ $\geqq C(A) \cdot \rho\left(\gamma_{1}^{u}\right) \cdot v_{0}(A)$, due to the homogeneity of the parallelogram $A$. Therefore, we also have $v_{\mathbf{E}}^{L}(A) \geqq C_{2}(A)>0$.

Remark. The proof of Lemma 13 requires the quadrilateral $\Pi$ to be small enough and $v_{0}(A(\Pi))>0$. However, we cannot state Lemma 13 and Corollary 14 for all quadrilaterals with such properties. Indeed, we have supposed the field $\mathbf{E}$ to be weak enough after choosing $\Pi$, i.e. actually we have required $|\mathbf{E}|<E_{0}(\Pi)$.

Let $A=A(\Pi)$ be a maximal parallelogram with the support $\Pi$ involved in Lemma 13. Consider a new map $T_{*}$ defined only on the fiber $\gamma^{u}$ and on the components of its images. This map is specified by an "absorbing" property of the parallelogram $A=A(\Pi)$. It acts exactly as the map $T_{\mathbf{E}}$ unless a component, $\gamma_{1}^{u}$, intersects both $s$-sides of the quadrilateral $\Pi$. In that last case the part $\gamma_{1}^{u} \cap A$ stops moving under $T_{*}$, and then all the future iterates of $T_{*}$ on that part are identities. The remaining part, i.e. $\gamma_{1}^{u} \backslash A$, consists of a countable number of curves - subcomponents - on which $T_{*}$ still acts as the map $T_{\mathbf{E}}$. After $n \geqq 1$ iterates of $T_{*}$, a part of $\gamma^{u}$ will be sooner or later "stuck" with the parallelogram $A$ while the remaining part of it will be still moving. We denote that remaining part by $\tilde{\gamma}^{u}(n)$. Obviously, its $p_{n}^{c}$-measure monotonically decreases in $n$.

The next lemma is a natural extension of Lemma 13. It was first introduced in [8] for piecewise linear toral maps. Its proof [8] is based on the hyperbolic properties of $T$ alone, so it works in our situation as well.

Lemma 15 (From long components into a fixed parallelogram). For any fiber $\gamma^{u}$ of length $\geqq D$ and any $n \geqq 1$ one has $p^{c}\left(\tilde{\gamma}^{u}(n)\right) \leqq c_{24} \alpha_{8}^{n}$, where $c_{24}>0$ and $\alpha_{8} \in(0,1)$ are constants, both independent of $\gamma^{u}$.

Roughly speaking, Lemmas 10,11 say that a short fiber is sufficiently fast transformed into long fibers, Lemma 13 says that a long fiber sufficiently fast sends some of its portions into fibers $u$-inscribed in $\Pi$, and Lemma 15 tells that a long fiber is sufficiently fast transformed under $T_{*}$ into Cantor sets lying on fibers $u$-inscribed in $\Pi$ and covering the points of $A(\Pi)$ on those fibers.

Remark. Our dynamics is obviously reversible. That is, our Lemmas $10-15$ have dual forms for stable fibers and negative powers of $T_{\mathbf{E}}$ (or, respectively, the iterates of a new map $T_{*}^{(-)}$which can be defined in a similar fashion as $T_{*}$ by the action of $T_{\mathbf{E}}^{-1}$ and an "absorbing" property of $A$ ).

The last remark actually provides the tool for the estimation of the values of $v_{\mathbf{E}}^{U, L}(B)$ for the arbitrary parallelogram $B$. First we describe the main idea of that estimation and then work out the details. Let $B$ be an arbitrary small parallelogram with $v_{0}(B)>0$, which is also homogeneous and maximal. Consider an arbitrary $y \in B$. By the dual statements to Lemmas $10-15$ the fiber $\gamma^{s}(y)$ is sufficiently fast transformed into $A$ under $T_{*}^{(-) n}, n \geqq 1$, see the above remark. Each time when a component of $T_{*}^{(-) n} \gamma^{s}(y)$ crosses both $u$-sides of $A$, it also intersects $A$ and the points of $T^{-n} B$ on that component cover all the points of $A$ on it, due to maximality of both $A$ and $B$. We can extend the definition of $T_{*}^{(-)}$to the whole parallelogram $B$ and its images under $T^{-n}, n \geqq 1$. This means that $T_{*}^{(-)}$acts exactly as $T_{\mathbf{E}}^{-1}$ unless a component of $T_{\mathbf{E}}^{-n} B$ crosses both $u$-sides of $\Pi(A)$. In that last case the intersection $T_{\mathbf{E}}^{-n} B \cap A$ stops, and on the remaining part, $T_{\mathbf{E}}^{-n} B \backslash A$, which consists of a countable number of parallelogarms - subcomponents - the map $T_{*}^{(-)}$will still act as 
$T_{\mathbf{E}}^{-1}$. Thus, by Lemmas $10-15$, the parallelogram $B$ itself is sufficiently fast transformed into $A$ under $T_{*}^{(-)}$.

Fix now a large $n_{0}=n_{0}(B)$ and consider the sets $B_{n}^{(-)}=T_{*}^{(-)\left(n-n_{0}\right)}\left(T_{\mathbf{E}}^{-n_{0}} B\right)$. In other words, $B_{n}^{(-)}, n \geqq 1$ are produced by the evolution of $B$ in the past when, during the first $n_{0}$ iterates only, it evolves "freely" under $T^{-1}$ and, then, the "absorbing" property of $A$ is turned on. The sets $B_{n}^{(-)}$for large $n>n_{0}$ then consist of a finite number of subparallelograms "stuck" with $A$ and of some parallelograms outside $A$ which are still moving under $T_{*}^{(-)}$. The preimages of the former are disjoint subparallelograms in $B$. Clearly, each of those preimages in transformed into $A$ by $T_{\mathbf{E}-k}$ for some $k>n_{0}$ and then its image under $T_{\mathbf{E}}^{-k}$ belongs either to $\mathfrak{R}\left(T^{-k} B \cap A\right)$ or to $\mathfrak{I}\left(T^{-k} B \cap A\right)$. We denote all the parallelograms of the first (regular) kind by $B_{1}, B_{2}, \ldots$ Each $B_{i}, i \geqq 1$ is a subparallelogram in $B$ and there is a $k_{i}>n_{0}$ such that $T^{-k_{i}} B_{i} \subset \mathfrak{R}\left(A \cap T^{-k_{i}} B\right)$. The parallelograms of the second (irregular) kind are less important for us and we denote their union by $B^{(0)}$.

Now we can estimate the measure $p_{N}^{c}(B)$ for large values of $N$. For each $i \geqq 1$ the parallelogram $T^{-k_{i} B_{i}}$ is $s$-inscribed in $A$, so that $p_{N-k_{i}}^{c}\left(T^{-k_{i}} B_{i}\right)$ is approximately $p_{N-k_{i}}^{c}(A) \cdot v_{0}\left(T^{-k_{i}} B_{i}\right) / v_{0}(A)$ due to (26), (27). This is an approximation with an exponentially small error, precisely

$$
\left|\frac{p_{N-k_{i}}^{c}\left(T^{-k_{i}} B_{i}\right) v_{0}(A)}{p_{N-k_{i}}^{c}(A) v_{0}\left(T^{-k_{i}} B_{i}\right)}-1\right| \leqq c_{25} \alpha_{9}^{m},
$$

where $m$ is the order of homogeneity of the parallelogram $A$, as in (27). We now obtain

$$
p_{N}^{c}\left(\bigcup B_{i}\right)=\sum_{i} p_{N-k_{\imath}}^{c}\left(T^{-k_{i}} B_{i}\right)=\left(1+\Delta_{m}\right)\left(v_{0}(A)\right)^{-1} \sum_{i} p_{N-k_{\imath}}^{c}(A) v_{0}\left(T^{-k_{\imath}} B_{i}\right) .
$$

The error term $\Delta_{m}$ here is exponentially small in $m$, as stated in (28).

Next we estimate the value $p_{N}^{c}\left(B \backslash \bigcup B_{i}\right)$. First, the $p_{N}^{c}$-measure of the set $\left(B \backslash\left(B^{(0)} \cup\left(\bigcup B_{i}\right)\right)\right)$ is exponentially small in $n_{1}-n_{0}$ due to Lemmas $10-15$, because that set consists of the points $y \in B$ such that $T^{-n_{1}} y, \ldots, T^{-n_{0}} y$ do not belong to $A$. The estimation of the value $p_{N}^{c}\left(B^{(0)}\right)$ is based on the following lemma.

Lemma 16 (Bound for irregular parts). $p_{N}^{c}\left(\mathfrak{I}\left(T^{-n} B \cap A\right)\right) \leqq c_{26} \alpha_{10}^{n}$ for certain $\alpha_{10} \in$ $(0,1)$ and all $N \geqq 1, n \geqq 1$.

The proof of Lemma 16 is essentially the same as that of Proposition 5.2 in [8]. The only difference is that we use here the measure $p_{N}^{c}$ instead of the invariant smooth measure on $M$. The validity of this change of measures is justified by our Remark 9.

Summarizing the above estimates, we obtain the decomposition

$$
p_{N}^{c}(B)=\left(1+\Delta_{m}\right)\left(v_{0}(A)\right)^{-1} \sum_{i} p_{N-k_{i}}^{c}(A) v_{0}\left(T^{-k_{i}} B_{i}\right)+\Delta_{0}+\Delta_{1}
$$

where $\left|\Delta_{1}\right|<c_{27} \alpha_{11}^{n_{1}-n_{0}}$ and $\left|\Delta_{0}\right|<c_{28} \alpha_{12}^{n_{0}}$ for certain $\alpha_{11}, \alpha_{12} \in(0,1)$.

The remarkable formula (29) allows us to estimate the measure $p_{N}^{c}(B)$ for an arbitrary homogeneous maximal parallelogram $B$. Denote $\varepsilon=v_{0}(B)$, then $\rho\left(\gamma_{B}^{s}(y)\right) \geqq c_{29} \varepsilon$ for any $y \in B$. Now we choose $n_{0}=-C_{0} \ln \varepsilon$ and $n_{1}=-C_{1} \ln \varepsilon$ with some large constants $C_{0}<C_{1}$. Then both $\left|\Delta_{0}\right|$ and $\left|\Delta_{1}\right|$ in (29) do not exceed $\varepsilon^{D}$ with some large $D>0$. On the other hand, if the difference $C_{1}-C_{0}$ is also large enough, then the majority of points of $B$ are transformed into $A$ under the map 


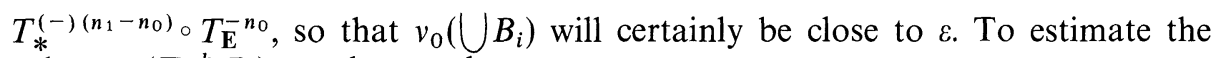
values $v_{0}\left(T^{-k_{i}} B_{i}\right)$ we observe that

$$
\ln \left(v_{0}\left(T^{-k_{2}} B_{i}\right) / v_{0}\left(B_{i}\right)\right) \leqq \omega|\mathbf{E}| n_{1}=-C_{1} \omega|\mathbf{E}| \ln \varepsilon
$$

due to (20). This implies the estimate

$$
\varepsilon^{1+C_{1} \omega|\mathbf{E}|} \leqq \sum_{i} \nu_{0}\left(T^{-k_{i}} B_{i}\right) \leqq \varepsilon^{1-C_{1} \omega|\mathbf{E}|} .
$$

We obtain for small $|\mathbf{E}|$ that the first term in the RHS of (29) is actually the principal one and we can neglect the others. It is also useful to note how the singularity of the limit measure $v_{\mathbf{E}}^{+}$can arise. The inequalities (30) imply

$$
\left(v_{0}(B)\right)^{C_{1} \omega|\mathbf{E}|} \leqq \frac{p_{N}^{c}(B)}{v_{0}(B)} \leqq\left(v_{0}(B)\right)^{-C_{1} \omega|\mathbf{E}|} .
$$

Thus the "density" of $p_{N}^{c}$ with respect to $v_{0}$ can approach either zero or infinity as $N \rightarrow \infty$ depending on which of two processes dominates: the contracting or the expanding. Due to (20) the contracting prevails when the particle with the initial conditions in $B$ travels mainly in the direction of the field $\mathbf{E}$. The expanding prevails when the particle travels in the opposite direction. The displacement of the particle in the perpendicular direction causes no effect on the density of $p_{N}^{c}$.

Remark. In our model the particle has enough freedom to travel along or opposite to the field direction. If the billiard table is closed or extended only in the perpendicular direction to the applied field, then the particle has no such freedom and the density of $p_{N}^{c}$ stays uniformly bounded. Although that density apparently oscillates as $N$ grows, the limit measure $v_{\mathbf{E}}^{+}$nevertheless exists and is absolutely continuous with respect to the Lebesgue measure. Our response theory (Propositions $1-4$ ) is formally correct but trivial since the main constants $\mathbf{D}, \boldsymbol{\sigma}$ and the current $\mathbf{J}$ are all zeroes.

As an immediate consequence of the decomposition (29) and the above remarks we obtain that $v_{\mathbf{E}}^{L}(B) \geqq C(B)>0$ as soon as $v_{0}(B)>0$. Here $C(B)$ is independent of the initial fiber $\gamma^{u}$ and of the field strength $\mathbf{E}$, provided the latter is small enough. Moreover, the decomposition (29) implies

$$
\frac{v_{\mathbf{E}}^{U}(B)}{v_{\mathbf{E}}^{L}(B)} \leqq\left(1+\Delta_{m}^{\prime}\right) \frac{v_{\mathbf{E}}^{U}(A)}{v_{\mathbf{E}}^{L}(A)},
$$

where the constant $\Delta_{m}^{\prime}$ is determined by $A$ alone and approaches zero as $m \rightarrow \infty$.

So far we have applied only "local" arguments studying the evolution of a particular parallelogram $B$. These have given us only a "rough" estimate (32). Next we are going to show that actually $v_{\mathbf{E}}^{U}(B)=v_{\mathbf{E}}^{L}(B)$ and thus this value determines $v_{\mathbf{E}}^{+}(B)$. To this end we have to involve certain "global" arguments. Namely, we use the Markov sieve $\mathbf{5}_{n}$ for some large $n$ and study the joint evolution of all its parallelograms. It can be well approximated by a probabilistic Markov chain as is explained below.

The properties of the Markov sieve $\mathfrak{G}_{n}$ and our Lemma 11 yield the bound

$$
p_{N}^{c}\left(\mathfrak{N}\left(\xi_{n}\right)\right) \leqq c_{30} \alpha_{13}^{n}
$$

for every large $N$, say, for $N \geqq-C \ln \rho\left(\gamma^{u}\right)$ for some large $C>0$. Furthermore, we can easily estimate the $p_{N}^{c}$-measure of the set of points in the quadrilaterals $\Pi_{1}, \ldots, \Pi_{I}$ which do not belong to the parallelograms $A_{1}, \ldots, A_{I}$. These points 
have too short unstable or stable fibers, so that Lemma 7, along with the above estimate (33), gives the bound

$$
p_{N}^{c}\left(M \backslash \bigcup A_{i}\right) \leqq c_{31} \alpha_{14}^{n} .
$$

In other words, the measure $p_{N}^{c}$ is almost concentrated on the Markov sieve $\mathfrak{G}_{n}$, up to an exponentially small error term.

Now denote $\pi_{i}(N)=p_{N}^{c}\left(A_{i}\right)$ and $\pi_{i j}^{(K)}(N)=p_{N+K}^{c}\left(A_{j} \cap T^{K} A_{i}\right) / p_{N}^{c}\left(A_{i}\right)$. Setting $A_{0}=M \backslash \bigcup A_{i}$ and letting the indices $i, j$ in the above notations run from 0 to $I$ we make $\left\|\pi_{i}(N)\right\|$ a probability distribution and $\left\|\pi_{i j}^{(K)}(N)\right\|$ a stochastic matrix. The measure $p_{N}^{c}$ inside the quadrilateral $\Pi_{i}$ is concentrated on a finite union of unstable fibers $u$-inscribed in $\Pi_{i}$ which are images of $\gamma^{u}$ under $T_{\mathbf{E}}^{n}$. Let $\tilde{\gamma}^{u}$ be one of those fibers and $\tilde{\gamma}_{i}^{u}=\tilde{\gamma}^{u} \cap A_{i}$. Denote $\tilde{p}^{c}$ the probabilistic conditional measure induced by $v_{0}$ on $\tilde{\gamma}_{i}^{u}$ and $\tilde{p}_{n}^{c}=T^{n} \tilde{p}^{c}$ for $n \geqq 1$. To the set $\tilde{\gamma}_{i}^{u}$ we can apply the above arguments involving a fixed parallelogram $A$ and resulting in the estimate (29). These arguments show again that

$$
\tilde{p}_{K}^{c}(B)=\left(1+\Delta_{m}\right)\left(v_{0}(A)\right)^{-1} \sum_{i} \tilde{p}_{K-k_{\mathbf{l}}}^{c}(A) v_{0}\left(T^{-k_{\imath}} B\right)+\Delta_{0}+\Delta_{1},
$$

where $B$ stands for $A_{j}$. Choose, as in (29), $n_{0}=C_{0} n, n_{1}=C_{1} n$ and $K=C_{2} n$ with sufficiently large constants $C_{0}, C_{1}, C_{2}$ such that $C_{1}-C_{0}$ and $C_{2}-C_{1}$ are also large enough. Then again both $\left|\Delta_{0}\right|$ and $\left|\Delta_{1}\right|$ in (35) do not exceed $\alpha_{5}^{n}$ for some small $c_{32} \alpha_{15}$ determined by $C_{0}$ and $C_{1}$.

Comparing (35) to (29) we conclude that $\tilde{p}_{K}^{c}(B) \geqq \frac{1}{2} p_{N}^{c}(B)$ provided $C_{0}, C_{1}$, $C_{2}$ and $N$ are large enough. As a result we obtain that

$$
\pi_{i j}^{(K)} \geqq \frac{1}{2} \pi_{j}(N)
$$

Next, due to the $n$-homogeneity of the parallelogram $A_{i}$ the values $\tilde{p}_{K}^{c}(B)$ are almost the same for different fibers $\tilde{\gamma}^{u} u$-inscribed in $\Pi_{i}$. To be specific, if $\tilde{\gamma}^{u}$ is another fiber of that kind, then

$$
\left|\tilde{\tilde{p}}_{K}^{c}(B) / \tilde{p}_{K}^{c}(B)-1\right| \leqq c_{33} \alpha_{16}^{n} .
$$

As a result, the values $\pi_{l j}^{(K)}(N)$ are almost independent of $N$, and so we can find an approximative stochastic matrix $\pi_{i j}^{(K)}$ such that

$$
\left|\pi_{i j}^{(K)}(N) / \pi_{i j}^{(K)}-1\right| \leqq c_{34} \alpha_{17}^{n}
$$

for all $i, j \geqq 1$. The estimate (37) yields also an important Markovian property

$$
\frac{p_{N+K}^{c}\left(A_{j} \cap T^{K} A_{i_{1}} \cap \ldots \cap T^{L K} A_{i_{L}}\right)}{p_{N}^{c}\left(A_{i_{1}} \cap \ldots \cap T^{(L-1) K} A_{i_{L}}\right)}=\pi_{i_{1} j}^{(K)}(N)\left(1+\Delta^{\prime}\right),
$$

where $\left|\Delta^{\prime}\right| \leqq c_{35} \alpha_{18}^{n}$. Moreover, $\pi_{i_{1} j}^{(K)}(N)$ in (39) can be replaced by $\pi_{i_{1} j}^{(K)}$ due to the approximation (38).

As a result we obtain an approximation of the joint evolution of the parallelograms of the Markov sieve $\mathfrak{6}_{n}$ by a stationary Markov chain. To be specific,

$$
\pi_{j}(N+L K)=(1+\Delta) \sum_{i_{1}, \ldots, i_{L}} \pi_{i_{L}}(N) \pi_{i_{L} i_{L-1}}^{(K)} \ldots \pi_{i_{1} j}^{(K)}
$$

with some $|\Delta|<c_{36} \alpha_{19}^{n}$, provided $L$ is not too large, say, $L=n$. Now we have an approximative stationary Markov chain (40) with the estimate (34) for the total 
measure of the "marginal" set $M \backslash \bigcup A_{i}$ and with the regularity condition (36) of Ibragimov type, see [21] and also [5]. These basic properties allow us to estimate the rate of mixing in the Markov chain and to prove a rapid convergence in $L$ of the probability distribution $\left\|\pi_{j}(N+L K)\right\|$ to the stationary distribution $\left\|\pi_{j}\right\|$ of the matrix $\left\|\pi_{i j}^{(K)}\right\|$. The corresponding reasonings involve typical estimates from the theory of Markov chains. The proof is essentially the same as that of Theorem 4.1 in [5], and we do not reproduce it here. The actual results are

$$
\sum_{j}\left|\pi_{j}(N+L K)-\pi_{j}\right| \leqq c_{37} \alpha_{20}^{n}
$$

and

$$
\sum_{j}\left|\frac{p_{N+L K}^{c}\left(T^{L K} A_{i} \cap A_{j}\right)}{\pi_{i}}-\pi_{j}\right| \leqq c_{38} \alpha_{21}^{n}
$$

for a typical parallelogram $A_{i}$. This last statement means that there is a subset $R_{*} \in \mathfrak{G}_{n}$ such that (42) holds for every $A_{i} \in R_{*}$, and the total $p_{N}^{c}$-measure of all the other parallelograms, i.e. those in $\mathfrak{G}_{n} \backslash R_{*}$, is less than $c_{39} \alpha_{22}^{n}$.

We are now able to prove the existence of the limit measure $v_{\mathbf{E}}^{+}$. First, for any quadrilateral $\Pi$ we prove that $v_{\mathbf{E}}^{U}(\Pi)=v_{\mathbf{E}}^{L}(\Pi)$. For large $n \geqq 1$ consider the Markov sieve $\mathfrak{G}_{n}$ with elements $A_{1}, \ldots, A_{2}$. The measure $v_{\mathbf{E}}^{U}\left(\Pi \backslash \bigcup A_{i}\right)$ is small enough due to (34). The parallelograms $A_{i}$ crossing the boundary $\partial \Pi$ also have a small total measure due to Remark 12. Therefore, the measures $\nu_{\mathbf{E}}^{U}$ and $\nu_{\mathbf{E}}^{L}$ are concentrated mainly on the union of parallelograms inside $\Pi$. We denote this union by $\Pi_{n}$. By virtue of (41) the measure $p_{N}^{c}\left(\Pi_{n}\right)$ is sufficiently close to the sum of the values $\pi_{i}$ for the parallelograms included into $\Pi_{n}$. Since this last sum is independent of $N$, we obtain that at least $v_{\mathbf{E}}^{U}(\Pi) / v_{\mathbf{E}}^{L}(\Pi) \leqq c_{40} \alpha_{23}^{n}$. Finally, $n$ here can be chosen arbitrarily large, so that actually $v_{\mathbf{E}}^{U}(\Pi)=v_{\mathbf{E}}^{\bar{L}}(\Pi)$.

These arguments can be also extended to maximal parallelograms. A maximal parallelogram $A$ can be obtained by removing from its support $\Pi(A)$ an infinite number of smaller quadrilaterals (gaps) $\Pi_{i}$, see e.g. $[4,5,8]$ for detail. The $v_{0}$ measures of those gaps decay exponentially fast, see e.g. [8, Lemma B.1]. Combining this fact with the estimate (31) we obtain the necessary tail bound for the $v_{\mathbf{E}}^{U}$ measures of those gaps, and then prove the formula $v_{\mathbf{E}}^{+}(A)=v_{\mathbf{E}}^{+}(\Pi(A))$ $-\sum_{i} v_{\mathbf{E}}^{+}\left(\Pi_{i}\right)$. Thus we establish the existence of the measure $v_{\mathbf{E}}^{+}$.

(e) Decay of Correlations. The estimate (42) has not yet been used. It readily yields the ergodicity of the measure $v_{\mathbf{E}}^{+}$. Moreover, we could as well establish a subexponential rate of the decay of correlations with respect to that measure, as for billiards in [5]. However, we do not need this exactly. What we really need in Sect. (a) is an estimate for the decay of correlations with respect to the measure $v_{0}$.

Let us fix here our definition of the Hölder classes $H_{\alpha}^{*}$, for small $|\mathbf{E}|$, by specifying the set of allowed discontinuity to be the singularity sets of the maps $T_{\mathbf{E}}^{ \pm}$and $T_{0}^{ \pm}$. Then, for instance, the functions $\tau_{0}(x)$ and $\Delta_{0}(x)$, as well as $\tau_{\mathbf{E}}(x)$ and $\Delta_{\mathbf{E}}(x)$, belong to $H_{\alpha}^{*}$ (see below).

Theorem 17 (Decay of correlations). For any two functions $f, g \in H_{\alpha}^{*}$ such that

$$
v_{0}(f)=0
$$

and for any $n \geqq 1$ we have

$$
\left|v_{0}\left(f \cdot\left(g \circ T_{\mathbf{E}}^{n}\right)\right)\right| \leqq C(f, g) \lambda_{1}^{\sqrt{n}},
$$

where $\lambda_{1} \in(0,1)$ is a constant determined by $T$ and $\alpha$. 
The proof of Theorem 17 is based on the estimate (42) along with supplementary estimates (34). It goes the same way as the proof of Theorem 1.1 in [5] and we omit the details.

We need also certain estimates for the constants $\lambda_{1}$ and $C(f, g)$ in Theorem 17 . These estimates readily come from the proof of Theorem 17 and were first explicitly given in [8]. The constant $\lambda_{1}$ can be chosen as $\lambda_{2}^{\alpha}$ for some $\lambda_{2} \in(0,1)$ which is independent of $\alpha$ and $\mathbf{E}$. Furthermore, we can set $C(f, g)=\left(C_{f}+M_{f}\right)\left(C_{g}+M_{g}\right)$, where $C_{f}$ is the factor in the Hölder condition and $M_{f}=\max _{M}|f(x)|$.

We now return to the specific function

$$
f_{\mathbf{E}}(x)=1-\exp \left[-\beta \mathbf{E} \cdot \Delta_{\mathbf{E}}(x)\right]
$$

which appears in Part I and Sect. (a). This function lies in a Hölder class $H_{\alpha}^{*}$ and, in fact, the corresponding coefficients $C_{f}, M_{f}$ vanish at least linearly in $\mathbf{E}$ as $\mathbf{E} \rightarrow 0$. To be specific,

$$
|f(x)| \leqq 2|\mathbf{E}| \beta \Delta_{\max },
$$

where $\Delta_{\max }=\max _{\mathbf{E}} \max _{x \in M}\left|\Delta_{\mathbf{E}}(x)\right|$ is finite due to the finiteness of the horizon. Furthermore if $x, y$ belong to the same component of smoothness of $T_{\mathbf{E}}$, then

$$
\left|f_{\mathbf{E}}(x)-f_{\mathbf{E}}(y)\right| \leqq 2|\mathbf{E}| \beta\left|\boldsymbol{\Delta}_{\mathbf{E}}(x)-\boldsymbol{\Delta}_{\mathbf{E}}(y)\right| \leqq 2|\mathbf{E}| \beta C|x-y|^{1 / 2},
$$

where $C>0$ is independent of $\mathbf{E}$. This last estimate is easy to check for $\mathbf{E}=0$, and then we apply the $C^{2}$ closeness of $T_{\mathbf{E}}$ to $T_{0}$ for small $|\mathbf{E}|$. The estimate (44) also gives the exponent $\alpha=1 / 2$ in the Hölder condition.

The estimates (43) and (44) give a uniform in $\mathbf{E}$ bound for the decay of correlations which we need in Sect. (a). Let $g_{\mathrm{E}}$ be another function in $H_{\alpha}^{*}$ also depending on $\mathbf{E}$ such that $C_{g}$ and $M_{g}$ are uniformly bounded in $\mathbf{E}$. Then

$$
\left|v_{0}\left(f_{\mathbf{E}} \cdot\left(g_{\mathbf{E}} \circ T_{\mathbf{E}}^{n}\right)\right)\right| \leqq|\mathbf{E}| C_{0}(f, g) \lambda_{2}^{\sqrt{n}},
$$

where $\lambda_{2} \in(0,1)$ does not depend on $\mathbf{E}$ and $C_{0}(f, g)$ is uniformly bounded in $\mathbf{E}$.

(f) Entropy and fractal dimension. In this last section we prove the parts of Proposition 1 concerning entropy and fractal dimension for our measure $v_{\mathbf{E}}^{+}$. $T_{\mathrm{E}}$ as

First, the Pesin formula expresses the measure-theoretic entropy of the map

$$
h_{\nu_{\mathbf{E}}^{+}}\left(T_{\mathbf{E}}\right)=\tilde{\lambda}_{\mathbf{E}}^{u}
$$

where $\tilde{\lambda}_{\mathbf{E}}^{u}$ is the positive Lyapunov exponent for $T_{\mathbf{E}}$, which is $v_{\mathbf{E}}^{+}$-a.e. constant in $M$ due to the ergodicity. This formula has been proved in [23] for hyperbolic maps with singularities with the only assumption that the underlying invariant measure is absolutely continuous on unstable fibers, which is true in our case. The entropy of the flow $S_{\mathbf{E}}^{t}$ in the full space $\mathfrak{M}$ is related to that of the map $T_{\mathbf{E}}$ through the well-kinown Abramov formula [1]:

$$
h_{\mu_{\mathbf{E}}}\left(\left\{S_{\mathbf{E}}^{1}\right\}\right)=\bar{\tau}_{\mathbf{E}}^{-1} h_{v_{\mathbf{E}}^{+}}\left(T_{\mathbf{E}}\right) .
$$

Note that another expression for the entropy of a hyperbolic map exists, which is in our notations

$$
h_{v_{\mathbf{E}}^{+}}\left(T_{\mathbf{E}}\right)=v_{\mathbf{E}}^{+}\left(\tilde{\Lambda}_{\mathbf{E}}^{u}\right)
$$

where $\tilde{\Lambda}_{\mathbf{E}}^{u}(x)$ is the local exponential rate of expansion for $x \in M$ under the map $T_{\mathbf{E}}$; see also (19). 
As $\mathbf{E} \rightarrow 0$, the function $\tilde{\Lambda}_{\mathbf{E}}^{u}(x)$ converges to $\tilde{\Lambda}_{0}^{u}(x)$ for every $x \in M \backslash\left(\bigcup S_{n}\right)$. Although that convergence is nonuniform, all those functions are uniformly bounded and continuous on their domains of definition. Furthermore, the measure $v_{\mathbf{E}}^{+}$weakly converges to $v_{0}$ as $\mathbf{E} \rightarrow 0$ due to our estimate (31). Hence the RHS of (45) converges to $v_{0}\left(\tilde{\Lambda}_{0}^{u}\right)$ as $\mathbf{E} \rightarrow 0$, so that

$$
\lim _{\mathbf{E} \rightarrow 0} h_{v_{\mathbf{E}}^{+}}\left(T_{\mathbf{E}}\right)=h_{v_{0}}\left(T_{0}\right) .
$$

This is what we needed for the Corollary to Propositions 1 and 4 in Part I.

Our estimates of the fractal dimension of the measure $\nu_{\mathbf{E}}^{+}$are based on the approach by L.-S. Young. In her paper [43] a chain of relations between the Hausdorff dimension $H D(v)$, the capacity $C(v)$, the Renyi dimension $R D(v)$ and the entropy $h(v)$ has been proven for an ergodic measure $v$ of a $C^{1+\alpha}$ diffeomorphism $T$ of a compact two-dimensional surface:

$$
H D(v)=C(v)=R D(v)=h_{v}(T)\left[\frac{1}{\tilde{\lambda}_{u}}-\frac{1}{\tilde{\lambda}_{s}}\right] .
$$

Note that $\tilde{\lambda}^{s}$ is negative, so that the expression in the brackets is always positive. Our map $T_{\mathbf{E}}$ is discontinuous, but its singularities are mild enough to extend the proofs of (46) to $T_{\mathbf{E}}$ along the lines of Katok-Strelcyn [23]. The modifications are minor and we do not go into detail.

Acknowledgements. N.C. and Ya.S. would like to thank L.-S. Young for the suggestion that her dimension formula may be applicable to our problem. G.E. and J.L.L. would like to thank W. Hoover, D.J. Evans, and E.G.D. Cohen for introducing us to the Gaussian method and its applications in non-equilibrium molecular dynamics. All the authors thank G. Gallavotti for his many very helpful discussions concerning various aspects of the whole subject. Finally, G.E. and J.L.L. wish to acknowledge the support of the Air Force Office of Scientific Research, Grant No. AF-0010E-91, for its support while completing this work.

\section{References}

1. Abramov, L.M.: On the Entropy of a Flow. Dokl. Akad. Nauk SSSR 128, 873-875 (1959)

2. Bunimovich, L.A., Sinai, Ya.G.: Markov Partitions for Dispersed Billiards. Commun. Math. Phys. 73, 247-280 (1980)

3. Bunimovich, L.A., Sinai, Ya.G.: Statistical Properties of Lorentz Gas with Periodic Configuration of Scatterers. Commun. Math. Phys. 78, 479-497 (1981)

4. Bunimovich, L.A., Sinai, Ya.G., Chernov, N.I.: Markov Partitions for Two-Dimensional Hyperbolic Billiards. Russ. Math. Surv. 45, 105-152 (1990)

5. Bunimovich, L.A., Sinai, Ya.G., Chernov, N.I.: Statistical Properties of Two-Dimensional Hyperbolic Billiards. Russ. Math. Surv. 46, 47-106 (1991)

6. Bunimovich, L.A.: A Theorem on Ergodicity of Two-Dimensional Hyperbolic Billiards. Commun. Math. Phys. 130, 599-621 (1990)

7. Chernov, N.I.: The Ergodicity of a Hamiltonian System of Two Particles in an External Field. Physica D 53, 233-239 (1991)

8. Chernov, N.I.: Ergodic and Statistical Properties of Piecewise Linear Hyperbolic Automorphisms of the 2-Torus. J. Stat. Phys. 69, 111-134 (1992)

9. Chernov, N.I.: Statistical Properties of the Periodic Lorentz Gas: Multidimensional Case. In preparation

10. Cornfeld, I.P., Fomin, S.V., Sinai, Ya.G.: Ergodic Theory. Berlin, Heidelberg, New York: Springer 1982 
11. Donnay, V., Liverani, C.: Potentials on the Two-Torus for Which the Hamiltonian Flow is Ergodic. Commun. Math. Phys. 135, 267-302 (1991)

12. Evans, D.J., Morriss, G.P.: Statistical Mechanics of Nonequilibrium Liquids. San Diego, CA: Academic Press 1990

13. Eyink, G.L., Lebowit, J.L., Spohn, H.: Microscopic Origin of Hydrodynamic Behavior: Entropy Production and the Steady State. Chaos/Xaoc. Soviet-American Perspectives on Nonlinear Science. New York: American Institute of Physics, 1990, pp. 367-391

14. Eyink, G.L., Lebowitz, J.L., Spohn, H.: Hydrodynamics of Stationary Non-Equilibrium States for Some Stochastic Lattice Gas Models. Commun. Math. Phys. 132, 253-283 (1990)

15. Gallavotti, G., Ornstein, D.: Billiards and Bernoulli schemes. Commun. Math. Phys. 38, 83-101 (1974)

16. Gauss, K.F.: Uber ein neues allgemeines Grundgesetz der Mechanik. J. Reine Angew. Math. IV, 232-235 (1829)

17. Goldstein, S., Kipnis, C., Ianiro, N.: Stationary States for a Mechanical System with Stochastic Boundary Conditions. J. Stat. Phys. 41, 915-939 (1985)

18. Goldstein, S., Lebowitz, J.L., Presutti, E.: Mechanical Systems with Stochastic Boundaries. Colloquia Mathematicae Societatis Janos Bolyai 27, Random Fields. Amsterdam: NorthHolland 1981

19. de Groot, S., Masur, P.: Nonequilibrium Thermodynamics. Amsterdam: North-Holland 1962

20. Hoover, W.G.: Computational Statistical Mechanics. Amsterdam; Elsevier 1991

21. Ibragimov, I.A., Linnik, Y.V.: Independent and Stationary Sequences of Random Variables. Gröningen: Wolters-Noordhoff 1971

22. van Kampen, N.: The Case Against Linear Response Theory. Physica Norvegica 5, 279-284 (1971)

23. Katok, A., Strelcyn, J.-M.: Invariant Manifolds, Entropy, and Billiards; Smooth Maps with Singularities. Lecture Notes in Mathematics, vol. 1222, New York: Springer 1986

24. Katz, S., Lebowitz, J.L., Spohn, H.: Nonequilibrium Steady States of Stochastic Lattice Gas Models of Fast Ionic Conductors. J. Stat. Phys. 34, 497-537 (1984)

25. Krámli, A., Simányi, N., Száss, D.: A "Transversal" Fundamental Theorem for Semi-Dispersing Billiards. Commun. Math. Phys. 129, 535-560 (1990)

26. Kubo, R.: Statistical Mechanical Theory of Irreversible Processes. I. J. Phys. Soc. Jap. 12, $570-586(1957)$

27. Lebowitz, J.L.: Stationary Nonequilibrium Gibbsian Ensembles. Phys. Rev. 114, 1192-1202 (1959)

28. Lebowitz, J.L., Bergmann, P.G.: Irreversible Gibbsian Ensembles. Ann. Phys. 1, 1-23 (1957)

29. McLennan, J.A. Jr.: Statistical Mechanics of the Steady State. Phys. Rev. 115, 1405-1409 (1959)

30. Moran, B., Hoover, W.: Diffusion in a Periodic Lorentz Gas. J. Stat. Phys. 48, 709-726 (1987)

31. Morris, G.P., Evans, D.J., Cohen, E.G.D., van Beijeren, H.: Phys. Rev. Lett. 62, 1579 (1989)

32. Ornstein, D.S., Weiss, B.: Statistical Properties of Chaotic Systems. Bull. Am. Math. Soc. 24, 11-116 (1991)

33. Ruelle, D.: Thermodynamic Formalism. New York: Addison-Wesley 1978

34. Sinai, Ya.G.: Dynamical Systems with Elastic Reflections. Ergodic Properties of Dispersing Billiards. Russ. Math. Surv. 25, 137-189 (1970)

35. Sinai, Ya.G., Chernov, N.I.: Ergodic Properties of some Systems of 2-Dimensional Discs and 3-Dimensional Spheres. Russ. Math. Surv. 42, 181-207 (1987)

36. Sinai, Ya.G.: Hyperbolic Billiards. Proceedings of the International Congress of Mathematicians, Kyoto, Japan, 1990

37. Toda, M., Kubo, R., Hashitume, N.: Statistical Physics II. Non-equilibrium Statistical Mechanics. Berlin, Heidelberg, New York: Springer 1985

38. Vaienti, S.: Ergodic Properties of the Discontinuous Sawtooth Map. J. Statist. Phys. 67 (1992) (to appear)

39. Vul, E.B., Sinai, Ya.G., Khanin, K.M.: Feigenbaum Universality and Thermodynamic Formalism. Russ. Math. Surv. 39, 1-40 (1984)

40. Wojtkowski, M.: Principles for the Design of Billiards with Nonvanishing Lyapunov Exponents. Commun. Math. Phys. 105, 391-414 (1986)

41. Yamada T., Kawasaki, K.: Nonlinear Effects in the Shear Viscosity of a Critical Mixture. Prog. Theor. Phys. 38, 1031-1051 (1967) 
42. Young, L.-S.: Bowen-Ruelle Measures for Certain Piecewise Hyperbolic Maps. Trans. Am. Math. Soc. 281, 41-48 (1985)

43. Young, L.-S.: Dimension, Entropy and Lyapunov Exponents. Erg. Th. and Dyn. Syst. 2, 109-124 (1982)

44. Zubarev, D.N.: The Statistical Operator for Nonequilibrium Systems. Sov. Phys. Dokl. 6, 776-778 (1962)

45. Zubarev, D.N.: Nonequilibrium Statistical Thermodynamics. New York: Consultants 1974.

46. Chernov, N.I., Eyink, G.L., Lebowitz, J.L., Sinai, Ya.G., Derivation of Ohm's Law in a Deterministic Mechanical Model. Submitted to Phys. Ref. Let.

Communicated by A. Jaffe 
